QUARTERLY OF APPLIED MATHEMATICS

VOLUME LXIV, NUMBER 2

JUNE 2006, PAGES 359-399

$\mathrm{S} 0033-569 \mathrm{X}(06) 01017-3$

Article electronically published on May 22, 2006

\title{
HOMOGENIZATION OF STRATIFIED THERMOVISCOPLASTIC MATERIALS
}

\author{
$\mathrm{BY}$ \\ NICOLAS CHARALAMBAKIS (Department of Civil Engineering, Aristotle University, \\ GR 54124 Thessaloniki, Greece) \\ AND \\ FRANÇOIS MURAT (Laboratoire Jacques-Louis Lions, Université Pierre et Marie Curie, \\ Bô̂te courrier 187, 75252 Paris Cedex 05, France)
}

Abstract. In the present paper we study the homogenization of the system of partial differential equations

$$
\begin{aligned}
& \rho^{\varepsilon}(x) \frac{\partial v^{\varepsilon}}{\partial t}-\frac{\partial}{\partial x}\left(\mu^{\varepsilon}\left(x, \theta^{\varepsilon}\right) \frac{\partial v^{\varepsilon}}{\partial x}\right)=f, \\
& c^{\varepsilon}\left(x, \theta^{\varepsilon}\right) \frac{\partial \theta^{\varepsilon}}{\partial t}=\mu^{\varepsilon}\left(x, \theta^{\varepsilon}\right)\left(\frac{\partial v^{\varepsilon}}{\partial x}\right)^{2},
\end{aligned}
$$

posed in $a<x<b, 0<t<T$, completed by boundary conditions on $v^{\varepsilon}$ and by initial conditions on $v^{\varepsilon}$ and $\theta^{\varepsilon}$. The unknowns are the velocity $v^{\varepsilon}$ and the temperature $\theta^{\varepsilon}$, while the coefficients $\rho^{\varepsilon}, \mu^{\varepsilon}$ and $c^{\varepsilon}$ are data which are assumed to satisfy

$$
\begin{gathered}
0<c_{1} \leq \mu^{\varepsilon}(x, s) \leq c_{2}, \quad 0<c_{3} \leq c^{\varepsilon}(x, s) \leq c_{4}, \quad 0<c_{5} \leq \rho^{\varepsilon}(x) \leq c_{6}, \\
-c_{7} \leq \frac{\partial \mu^{\varepsilon}}{\partial s}(x, s) \leq 0, \quad\left|c^{\varepsilon}(x, s)-c^{\varepsilon}\left(x, s^{\prime}\right)\right| \leq \omega\left(\left|s-s^{\prime}\right|\right) .
\end{gathered}
$$

This sequence of one-dimensional systems is a model for the homogenization of nonhomogeneous, stratified, thermoviscoplastic materials exhibiting thermal softening and a temperature-dependent rate of plastic work converted into heat.

Under the above hypotheses we prove that this system is stable by homogenization. More precisely one can extract a subsequence $\varepsilon^{\prime}$ for which the velocity $v^{\varepsilon^{\prime}}$ and the temperature $\theta^{\varepsilon^{\prime}}$ converge to some homogenized velocity $v^{0}$ and some homogenized temperature $\theta^{0}$ which solve a system similar to the system solved by $v^{\varepsilon}$ and $\theta^{\varepsilon}$, for coefficients $\rho^{0}$, $\mu^{0}$ and $c^{0}$ which satisfy hypotheses similar to the hypotheses satisfied by $\rho^{\varepsilon}, \mu^{\varepsilon}$ and $c^{\varepsilon}$.

Received December 1, 2005.

2000 Mathematics Subject Classification. Primary 74Q15, 74Q10, 35B27; Secondary 74C10, 74F05, 35Q72, 35M20, 35K55.

Key words and phrases. Homogenization, thermoviscoplastic materials.

E-mail address: charalam@civil.auth.gr

E-mail address: murat@ann.jussieu.fr

(C)2006 Brown University Reverts to public domain 28 years from publication 
These homogenized coefficients $\rho^{0}, \mu^{0}$ and $c^{0}$ are given by some explicit (even if sophisticated) formulas. In particular, the homogenized heat coefficient $c^{0}$ in general depends on the temperature even if the heterogeneous heat coefficients $c^{\varepsilon}$ do not depend on it.

Résumé. Dans cet article, nous étudions l'homogénéisation du système d'équations aux dérivées partielles

$$
\begin{aligned}
& \rho^{\varepsilon}(x) \frac{\partial v^{\varepsilon}}{\partial t}-\frac{\partial}{\partial x}\left(\mu^{\varepsilon}\left(x, \theta^{\varepsilon}\right) \frac{\partial v^{\varepsilon}}{\partial x}\right)=f, \\
& c^{\varepsilon}\left(x, \theta^{\varepsilon}\right) \frac{\partial \theta^{\varepsilon}}{\partial t}=\mu^{\varepsilon}\left(x, \theta^{\varepsilon}\right)\left(\frac{\partial v^{\varepsilon}}{\partial x}\right)^{2},
\end{aligned}
$$

posé dans $a<x<b, 0<t<T$ et complété par des conditions aux limites sur $v^{\varepsilon}$ et des conditions initiales sur $v^{\varepsilon}$ et $\theta^{\varepsilon}$. Les inconnues sont la vitesse $v^{\varepsilon}$ et la température $\theta^{\varepsilon}$, alors que les coefficients $\rho^{\varepsilon}, \mu^{\varepsilon}$ et $c^{\varepsilon}$ sont des données qui vérifient

$$
\begin{gathered}
0<c_{1} \leq \mu^{\varepsilon}(x, s) \leq c_{2}, \quad 0<c_{3} \leq c^{\varepsilon}(x, s) \leq c_{4}, \quad 0<c_{5} \leq \rho^{\varepsilon}(x) \leq c_{6}, \\
-c_{7} \leq \frac{\partial \mu^{\varepsilon}}{\partial s}(x, s) \leq 0, \quad\left|c^{\varepsilon}(x, s)-c^{\varepsilon}\left(x, s^{\prime}\right)\right| \leq \omega\left(\left|s-s^{\prime}\right|\right) .
\end{gathered}
$$

Cette suite de problèmes unidimensionnels modélise l'homogénéisation de matériaux thermoviscoplastiques hétérogènes dont la résistance diminue avec la température et dont le taux de travail plastique converti en chaleur dépend de la température.

Sous les hypothèses ci-dessus, nous démontrons que ce système est stable par homogénéisation. Plus précisément, on peut extraire une sous-suite $\varepsilon^{\prime}$ pour laquelle la vitesse $v^{\varepsilon^{\prime}}$ et la température $\theta^{\varepsilon^{\prime}}$ convergent vers une vitesse homogénéisée $v^{0}$ et une température homogénéisée $\theta^{0}$ qui sont solution d'un système similaire à celui dont $v^{\varepsilon}$ et $\theta^{\varepsilon}$ sont solution, pour des coefficients $\rho^{0}, \mu^{0}$ et $c^{0}$ qui satisfont des hypothèses analogues à celles satisfaites par $\rho^{\varepsilon}, \mu^{\varepsilon}$ et $c^{\varepsilon}$. Les coefficients homogénéisés $\rho^{0}, \mu^{0}$ et $c^{0}$ sont donnés par des formules explicites (même si elles sont assez compliquées). En particulier le coefficient thermique homogénéisé $c^{0}$ dépend en général de la température, même si les coefficients thermiques hétérogènes $c^{\varepsilon}$ n'en dépendent pas.



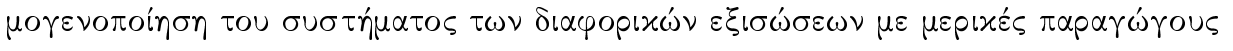

$$
\begin{gathered}
\rho^{\varepsilon}(x) \frac{\partial v^{\varepsilon}}{\partial t}-\frac{\partial}{\partial x}\left(\mu^{\varepsilon}\left(x, \theta^{\varepsilon}\right) \frac{\partial v^{\varepsilon}}{\partial x}\right)=f, \\
c^{\varepsilon}\left(x, \theta^{\varepsilon}\right) \frac{\partial \theta^{\varepsilon}}{\partial t}=\mu^{\varepsilon}\left(x, \theta^{\varepsilon}\right)\left(\frac{\partial v^{\varepsilon}}{\partial x}\right)^{2},
\end{gathered}
$$



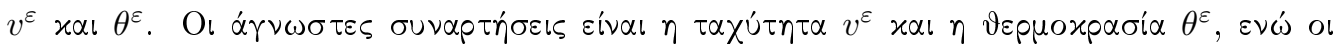

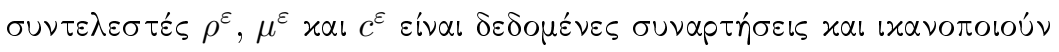

$$
\begin{gathered}
0<c_{1} \leq \mu^{\varepsilon}(x, s) \leq c_{2}, \quad 0<c_{3} \leq c^{\varepsilon}(x, s) \leq c_{4}, \quad 0<c_{5} \leq \rho^{\varepsilon}(x) \leq c_{6}, \\
-c_{7} \leq \frac{\partial \mu^{\varepsilon}}{\partial s}(x, s) \leq 0, \quad\left|c^{\varepsilon}(x, s)-c^{\varepsilon}\left(x, s^{\prime}\right)\right| \leq \omega\left(\left|s-s^{\prime}\right|\right) .
\end{gathered}
$$






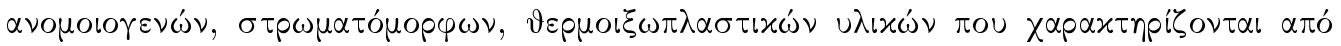



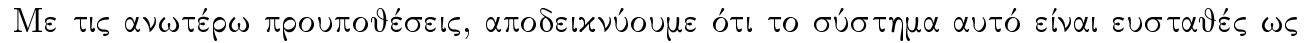

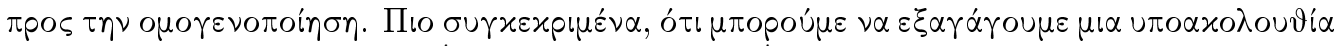



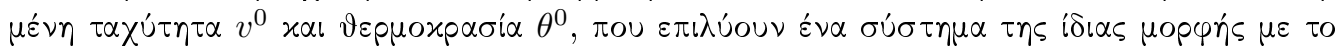







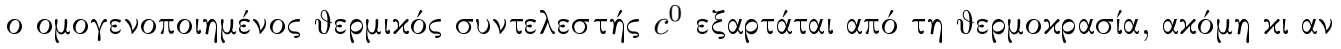

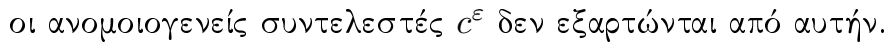

\section{Contents}

1. Introduction

2. Setting of the problem and homogenization result

2.1. Hypotheses

2.2. Existence, uniqueness and continuity with respect to the data result

2.3. Regularity results

2.4. Homogenization result

3. Definition of the homogenized viscosity function $\mu^{0}$ and of the homogenized heat coefficient $c^{0}$

3.1. Transformation of the problem

3.2. Definition of the homogenized functions $\mu^{0}$ and $c^{0}$

3.3. Proofs of Propositions 3.3 and 3.4

4. Proof of the regularity Theorem 2.4

5. Proof of the homogenization Theorem 2.5

5.1. First case: the case where $\frac{\partial f^{\varepsilon}}{\partial t}$ is bounded in $L^{2}\left(0, T ; H^{-1}(\Omega)\right)$

5.2. Second case: the case where $f^{\varepsilon}$ is compact in $L^{2}(Q)$

6. An example showing that the homogenized heat coefficient $c^{0}$ depends in general on the temperature

Acknowledgements

References

1. Introduction. In the present work we consider the homogenization of nonhomogeneous, stratified, thermoviscoplastic materials exhibiting thermal softening which are subjected to simple shearing. In mathematical terms we consider the system of partial differential equations (posed in the given one-dimensional space interval $(a, b), a<b$, 
and in the given time interval $(0, T), T>0)$

$$
\begin{gathered}
\rho^{\varepsilon}(x) \frac{\partial v^{\varepsilon}}{\partial t}=\frac{\partial \sigma^{\varepsilon}}{\partial x}+f(t, x) \quad \text { in }(0, T) \times(a, b), \\
c^{\varepsilon}\left(x, \theta^{\varepsilon}\right) \frac{\partial \theta^{\varepsilon}}{\partial t}=\sigma^{\varepsilon} \frac{\partial v^{\varepsilon}}{\partial x} \quad \text { in }(0, T) \times(a, b), \\
\sigma^{\varepsilon}=\mu^{\varepsilon}\left(x, \theta^{\varepsilon}\right) \frac{\partial v^{\varepsilon}}{\partial x} \quad \text { in }(0, T) \times(a, b),
\end{gathered}
$$

where the unknowns are the velocity $v^{\varepsilon}$, the temperature $\theta^{\varepsilon}$ and the stress $\sigma^{\varepsilon}$, while the data are the density $\rho^{\varepsilon}(x)$, the inertial forces $f(t, x)$, the heat coefficient $c^{\varepsilon}(x, s)$ and the viscosity function $\mu^{\varepsilon}(x, s)$. The system (1.1) -(1.3) has to be completed by initial conditions on $v^{\varepsilon}$ and $\theta^{\varepsilon}$, namely

$$
v^{\varepsilon}(0, x)=v_{0}^{\varepsilon}(x), \quad \theta^{\varepsilon}(0, x)=\theta_{0}^{\varepsilon}(x) \quad \text { in }(a, b),
$$

where $v_{0}^{\varepsilon}(x)$ and $\theta_{0}^{\varepsilon}(x)$ are given, and by boundary conditions which impose the shearing, namely either

$$
v^{\varepsilon}(t, a)=v_{a}^{\varepsilon}(t), \quad v^{\varepsilon}(t, b)=v_{b}^{\varepsilon}(t) \quad \text { in }(0, T),
$$

where $v_{a}^{\varepsilon}(t)$ and $v_{b}^{\varepsilon}(t)$ are given, when the boundary velocities are imposed (Dirichlet boundary conditions), or

$$
\sigma^{\varepsilon}(t, a)=\sigma_{a}^{\varepsilon}(t), \quad \sigma^{\varepsilon}(t, b)=\sigma_{b}^{\varepsilon}(t) \quad \text { in }(0, T),
$$

where $\sigma_{a}^{\varepsilon}(t)$ and $\sigma_{b}^{\varepsilon}(t)$ are given, when the boundary stresses are imposed (Neumann boundary conditions), or finally

$$
v^{\varepsilon}(t, a)=v_{a}^{\varepsilon}(t), \quad \sigma^{\varepsilon}(t, b)=\sigma_{b}^{\varepsilon}(t) \quad \text { in }(0, T),
$$

where $v_{a}^{\varepsilon}(t)$ and $\sigma_{b}^{\varepsilon}(t)$ are given, when the velocity is imposed in $x=a$ and the stress is imposed in $x=b$ (mixed boundary conditions).

The main features of the data are first the fact that $\rho^{\varepsilon}, c^{\varepsilon}$ and $\mu^{\varepsilon}$ are bounded from above and from below by strictly positive constants, second the fact that the functions $c^{\varepsilon}$ are uniformly (in $x$ and $\varepsilon$ ) continuous with respect to $s$, and that the functions $\mu^{\varepsilon}$ are uniformly (in $x$ and $\varepsilon$ ) Lipschitz continuous and nonincreasing with respect to $s$, and third the fact that all the functions $\rho^{\varepsilon}, c^{\varepsilon}$ and $\mu^{\varepsilon}$ depend on $x$ in a measurable (and not necessarily continuous 1 ) way. The latest property means that the materials under consideration are heterogeneous. Homogenization consists in studying the limit of the problems (1.1)-(1.3) when $\varepsilon$, which represents the typical size of heterogeneities, tends to zero.

The material described by (1.1) - (1.3) is stratified in the sense that it is made of layers perpendicular to a given direction of space, here the $x$ direction, while the shearing excerced by the boundary conditions is perpendicular to that direction, which allows one to reduce to one-dimensional (in space) partial differential equations. Homogenization

\footnotetext{
${ }^{1}$ Ten years ago, a new generation of heterogeneous materials, called functionally graded materials, appeared in the mechanical literature (see e.g. 1], 2], 3], 4] and 11]). These materials, characterized by high resistance to loading and/or to temperature increase, have been studied intensively, and numerical homogenization formulas have been proposed under the assumption of smoothly varying fields, which means that the different components of these materials are supposed to be perfectly bonded by thermomechanical processing in order to exhibit continuously changing properties. In contrast, our analysis does not assume any continuity of the coefficients with respect to the variable $x$.
} 
then consists in considering a (very) large number of (very) fine layers (of thickness, say, $\varepsilon$ ) of different materials, and in describing the overall behaviour of the velocity $v^{\varepsilon}$, temperature $\theta^{\varepsilon}$ and stress $\sigma^{\varepsilon}$ for fixed external forces. In mathematical terms, this overall behaviour is expressed by the weak limits $v^{0}, \theta^{0}$ and $\sigma^{0}$ of $v^{\varepsilon}, \theta^{\varepsilon}$ and $\sigma^{\varepsilon}$ as $\varepsilon$ tends to zero.

We prove in the present paper that $v^{0}, \theta^{0}$ and $\sigma^{0}$ satisfy a system of equations of the type (1.1)-(1.3), corresponding to coefficients $\rho^{0}(x), c^{0}(x, s)$ and $\mu^{0}(x, s)$. This is the main result of the present paper. It means that system (1.1)-(1.3) is stable under homogenization. In this process, the homogenized density $\rho^{0}$ is obtained as the weakstar limit of $\rho^{\varepsilon}$, but this is not the case of the homogenized heat coefficient $c^{0}(x, s)$ and of the homogenized viscosity function $\mu^{0}(x, s)$, which have to be defined through a much more sophisticated process (see Remark 3.5, where this process is summarized). In particular, a rather strange phenomenon occurs as far as $c^{\varepsilon}$ and $c^{0}$ are concerned. Even if in problem (1.1)-(1.3) the heat coefficient $c^{\varepsilon}(x, s)$ does not depend on $s$, i.e. even if $c^{\varepsilon}(x, s)=c^{\varepsilon}(x)$, the homogenized heat coefficient $c^{0}(x, s)$ does depend in general on $s$ (see Theorem 6.1, where explicit formulas are given in the case of a material made of layers of some given homogeneous phases). This mathematical result is in accordance with recent experiments and theoretical mechanical studies based on a temperaturedependent fraction of plastic work converted into heating (see [10, 12] and [13]). Indeed the heat coefficient $c$ is given by $c=\frac{\beta}{\rho h}$, where $h$ is the specific heat coefficient and $\beta$ the rate of plastic work converted into heating, a quantity which is related to the rearrangement of crystals during deformation. It is in general assumed in the literature that $\beta=0.9$. In particular, $\beta$ is constant with respect to the temperature $\theta$. If $\rho$ and $h$ are assumed to be constant with respect to $\theta$, which is a realistic assumption, then $c$ does not depend on $\theta$. Since our result shows that $c$ depends on $\theta$ for homogenized materials, the hypothesis $\beta=0.9$ is unrealistic. As experimental measurement of $\beta$ for different temperatures is very difficult, estimating $c$ may be very useful for understanding the overall behavior of stratified materials under high strain rates.

Homogenization is now a well-established mathematical theory, at least as elliptic or parabolic partial differential equations are concerned. We will only refer to the method of Tartar [15, and also, for general references, to the books of Sanchez-Palencia 14 . and of Bensoussan, Lions and Papanicolaou [6], even if those two books are concerned with the special case of periodic coefficients, a hypothesis which is not assumed in the present work. Let us explicitly observe that in the one-dimensional (in space) case, the homogenized coefficients can in general be expressed by formulas involving the weak limits of the heterogeneous coefficients, or more exactly of some (nonlinear) functions of them. Such is the case in the present paper.

However, from the bibliographical standpoint, to the best of our knowledge, there is no paper studying the homogenization of thermoviscoplastic materials, even if the existence of solutions for (1.1)-(1.3) has often been studied in the literature. As far as existence of a solution is concerned, we just quote the pioneering work of Dafermos and Hsiao [9, the works of Tzavaras [16] and [17, the recent paper [5] which is concerned with the numerical analysis of the problem, and our papers [7] and [8] (the second one 
presents existence and uniqueness results of the weak solution of (1.1)-(1.3), as well as its approximation by finite elements).

The plan of the paper is as follows.

In Section 2, we give the precise hypotheses under which we study the problem (Subsection 2.1), then we recall a result of existence, uniqueness and continuity with respect to the data (Subsection 2.2), and we state regularity results (Subsection 2.3).

Subsection 2.4 is devoted to the statement of the homogenization result (Theorem 2.5), which is the main result of the present paper.

The rest of the paper is mainly concerned with the proof of this theorem. We begin in Section 3 by defining the homogenized viscosity function $\mu^{0}$ and the homogenized heat coefficient $c^{0}$ (Subsection 3.2, see in particular Remark 3.5). Before of that, we motivate these definitions by a change of unknown functions, which transforms the problem in an equivalent, but simpler one (Subsection 3.1). The proofs concerned with the definitions of the homogenized viscosity function $\mu^{0}$ and heat coefficient $c^{0}$ are given in Subsection 3.3 .

We then pass in Section 4 to the proof of the regularity (in time) result stated in Subsection 2.3, and then in Section 5 to the proof the homogenization result.

Finally Section [6] is devoted to the study of the model example in which a heterogeneous material is made of fine layers (of size $\varepsilon$ ) of some homogeneous given phases. In this case we are able to give explicit formulas (Theorem 6.1) for the homogenized density $\rho^{0}$, the homogenized heat coefficient $c^{0}$ and the homogenized viscosity function $\mu^{0}$. These explicit formulas show that in general the homogenized heat coefficient $c^{0}$ depends on the temperature, even if the heat coefficients of the various phases do not depend on it.

\section{Setting of the problem and homogenization result.}

2.1. Hypotheses. In this entire paper, we consider $a, b$ and $T$ in $\mathbf{R}$ with $a<b$ and $T>0$, and we set

$$
(a, b)=\Omega, \quad(0, T) \times(a, b)=Q .
$$

We also consider a sequence of strictly positive numbers $\varepsilon$ which tend to zero.

We consider a sequence of Carathéodory functions $\mu^{\varepsilon}: \Omega \times \mathbf{R} \rightarrow \mathbf{R}$ (the viscosity functions) which are nonincreasing in $s$ and uniformly (in $x$ and $\varepsilon$ ) Lipschitz continuous in $s$, i.e.

$$
\left\{\begin{array}{l}
x \rightarrow \mu^{\varepsilon}(x, s) \text { is measurable } \forall s \in \mathbf{R}, \\
-c_{7}\left(s-s^{\prime}\right) \leq \mu^{\varepsilon}(x, s)-\mu^{\varepsilon}\left(x, s^{\prime}\right) \leq 0 \quad \text { a.e. } x \in \Omega, \forall s, s^{\prime} \in \mathbf{R}, s>s^{\prime},
\end{array}\right.
$$

where $c_{7}>0$ is a given constant. We also assume that

$$
c_{1} \leq \mu^{\varepsilon}(x, s) \leq c_{2} \quad \text { a.e. } x \in \Omega, \forall s \in \mathbf{R}
$$

where $0<c_{1} \leq c_{2}<+\infty$ are given constants. 
We also consider a sequence of Carathéodory functions $c^{\varepsilon}: \Omega \times \mathbf{R} \rightarrow \mathbf{R}$ (the heat coefficients) which are uniformly (in $x$ and $\varepsilon$ ) continuous in $s$, i.e.

$$
\left\{\begin{array}{l}
x \rightarrow c^{\varepsilon}(x, s) \text { is measurable } \forall s \in \mathbf{R}, \\
\left|c^{\varepsilon}(x, s)-c^{\varepsilon}\left(x, s^{\prime}\right)\right| \leq \omega\left(\left|s-s^{\prime}\right|\right) \quad \text { a.e. } x \in \Omega, \forall s, s^{\prime} \in \mathbf{R},
\end{array}\right.
$$

where $\omega$ is a modulus of continuity, i.e. a nondecreasing continuous function $\omega: \mathbf{R}^{+} \rightarrow \mathbf{R}^{+}$ with $\omega(0)=0$. We also assume that

$$
c_{3} \leq c^{\varepsilon}(x, s) \leq c_{4} \quad \text { a.e. } x \in \Omega, \forall s \in \mathbf{R},
$$

where $0<c_{3} \leq c_{4}<+\infty$ are given constants.

We finally consider a sequence of $L^{\infty}(\Omega)$ functions $\rho^{\varepsilon}$ (the densities). We assume that

$$
c_{5} \leq \rho_{\varepsilon}(x) \leq c_{6} \quad \text { a.e. } x \in \Omega,
$$

where $0<c_{5} \leq c_{6}<+\infty$ are given constants, and that

$$
\rho^{\varepsilon} \rightarrow \rho^{0} \quad \text { in } L^{\infty}(\Omega) \text { weak-star. }
$$

On the other hand, we consider sequences of external forces $f^{\varepsilon}$, of Dirichlet boundary conditions $v_{a}^{\varepsilon}$ and $v_{b}^{\varepsilon}$, and of initial conditions $v_{0}^{\varepsilon}$ and $\theta_{0}^{\varepsilon}$, and their limits $f^{0}, v_{a}^{0}, v_{b}^{0}, v_{0}^{0}$ and $\theta_{0}^{0}$, which satisfy for a given constant $K>0$

$$
\begin{gathered}
\left\{\begin{array}{l}
f^{\varepsilon} \in L^{2}(Q), \quad f^{0} \in L^{2}(Q), \quad\left\|f^{\varepsilon}\right\|_{L^{2}(Q) \leq K,} \\
f^{\varepsilon} \rightarrow f^{0} \quad \text { in } L^{2}(Q) \text { weak, }
\end{array}\right. \\
\left\{\begin{array}{l}
v_{a}^{\varepsilon} \in H^{1}(0, T), \quad v_{b}^{\varepsilon} \in H^{1}(0, T), \quad v_{a}^{0} \in H^{1}(0, T), \quad v_{b}^{0} \in H^{1}(0, T), \\
\left\|v_{a}^{\varepsilon}\right\|_{H^{1}(0, T)} \leq K, \quad\left\|v_{b}^{\varepsilon}\right\|_{H^{1}(0, T)} \leq K, \\
v_{a}^{\varepsilon} \rightarrow v_{a}^{0} \quad \text { in } H^{1}(0, T) \text { weak, } \quad v_{b}^{\varepsilon} \rightarrow v_{b}^{0} \quad \text { in } H^{1}(0, T) \text { weak, }
\end{array}\right. \\
\left\{\begin{array}{c}
v_{0}^{\varepsilon} \in H^{1}(\Omega), \quad v_{0}^{0} \in H^{1}(\Omega), \quad\left\|v_{0}^{\varepsilon}\right\|_{H^{1}(\Omega)} \leq K, \\
v_{0}^{\varepsilon} \rightarrow v_{0}^{0} \quad \text { in } H^{1}(\Omega) \text { weak, }, \\
v_{0}^{\varepsilon}(a)=v_{a}^{\varepsilon}(0), \quad v_{0}^{\varepsilon}(b)=v_{b}^{\varepsilon}(0),
\end{array}\right. \\
\left\{\begin{array}{c}
\theta_{0}^{\varepsilon} \in L^{1}(\Omega), \quad \theta_{0}^{0} \in L^{1}(\Omega), \quad \gamma^{0} \in L^{1}(\Omega), \quad\left\|\theta_{0}^{\varepsilon}\right\|_{L^{1}(\Omega)} \leq K, \\
\theta_{0}^{\varepsilon} \rightarrow \theta_{0}^{0} \quad \text { in } \mathcal{D}^{\prime}(\Omega) \text { weak-star, } \\
\mid \theta_{0}^{\varepsilon} \rightarrow \rightarrow \gamma^{0} \quad \text { in } \mathcal{D}^{\prime}(\Omega) \text { weak-star. }
\end{array}\right.
\end{gathered}
$$

Observe that (2.10) are compatibility conditions between the boundary values and the initial value of $v^{\varepsilon}$.

Observe also that introducing $\gamma^{0}$ in hypothesis (2.11) is not necessary when $\theta_{0}^{\varepsilon} \geq 0$, which is the physical case, since one has $\gamma^{0}=\theta_{0}^{0}$ in this case. The assumption $\gamma^{0} \in L^{1}(\Omega)$ will be used in (and only in) the proof of Proposition 3.3 below. 
2.2. Existence, uniqueness and continuity with respect to the data result. Theorems 2.1 and 3.1 of our paper [8] prove the following result of existence, uniqueness and local Lipschitz continuity with respect to the data for the case of Dirichlet boundary conditions (1.5) (see also [7] for another proof of the existence result).

TheOREm 2.1 (Existence, uniqueness and local Lipschitz continuity for Dirichlet boundary conditions). Assume that hypotheses (2.1) (2.11) hold true. Then there exists a unique couple $\left(v^{\varepsilon}, \theta^{\varepsilon}\right)$ which satisfies

$$
\begin{gathered}
v^{\varepsilon} \in L^{\infty}\left(0, T ; H^{1}(\Omega)\right) \cap H^{1}\left(0, T ; L^{2}(\Omega)\right), \\
\theta^{\varepsilon} \in W^{1,1}\left(0, T ; L^{1}(\Omega)\right), \\
\rho^{\varepsilon}(x) \frac{\partial v^{\varepsilon}}{\partial t}-\frac{\partial}{\partial x}\left(\mu^{\varepsilon}\left(x, \theta^{\varepsilon}\right) \frac{\partial v^{\varepsilon}}{\partial x}\right)=f^{\varepsilon} \quad \text { in } \mathcal{D}^{\prime}(Q), \\
c^{\varepsilon}\left(x, \theta^{\varepsilon}\right) \frac{\partial \theta^{\varepsilon}}{\partial t}=\mu^{\varepsilon}\left(x, \theta^{\varepsilon}\right)\left(\frac{\partial v^{\varepsilon}}{\partial x}\right)^{2} \quad \text { in } \mathcal{D}^{\prime}(Q), \\
v^{\varepsilon}(t, a)=v_{a}^{\varepsilon}(t), \quad v^{\varepsilon}(t, b)=v_{b}^{\varepsilon}(t) \quad \text { a.e. } t \in(0, T), \\
v^{\varepsilon}(0, x)=v_{0}^{\varepsilon}(x) \quad \text { a.e. } x \in \Omega, \\
\theta^{\varepsilon}(0, x)=\theta_{0}^{\varepsilon}(x) \quad \text { a.e. } x \in \Omega .
\end{gathered}
$$

Moreover the stress $\sigma^{\varepsilon}$ defined by

$$
\sigma^{\varepsilon}=\mu^{\varepsilon}\left(x, \theta^{\varepsilon}\right) \frac{\partial v^{\varepsilon}}{\partial x}
$$

satisfies

$$
\sigma^{\varepsilon} \in L^{\infty}\left(0, T ; L^{2}(\Omega)\right) \cap L^{2}\left(0, T ; H^{1}(\Omega)\right),
$$

and the following a priori estimates hold true

$$
\begin{gathered}
\left\|v^{\varepsilon}\right\|_{L^{\infty}\left(0, T ; H^{1}(\Omega)\right)}+\left\|\frac{\partial v^{\varepsilon}}{\partial t}\right\|_{L^{2}\left(0, T ; L^{2}(\Omega)\right)} \leq C^{\star} \\
\left\|\theta^{\varepsilon}\right\|_{L^{\infty}\left(0, T ; L^{1}(\Omega)\right)}+\left\|\frac{\partial \theta^{\varepsilon}}{\partial t}\right\|_{L^{1}\left(0, T ; L^{1}(\Omega)\right)} \leq C^{\star} \\
\left\|\sigma^{\varepsilon}\right\|_{L^{\infty}\left(0, T ; L^{2}(\Omega)\right)}+\left\|\sigma^{\varepsilon}\right\|_{L^{2}\left(0, T ; H^{1}(\Omega)\right)} \leq C^{\star}
\end{gathered}
$$

where $C^{\star}$ denotes a constant which depends only on $c_{1}, c_{2}, c_{3}, c_{4}, c_{5}, c_{6}$ and $K$.

Finally, if $\left(v^{\varepsilon}, \theta^{\varepsilon}\right)$ and $\left(\hat{v}^{\varepsilon}, \hat{\theta}^{\varepsilon}\right)$ are the (unique) solutions of (2.12)-(2.18) for the data $\left(f^{\varepsilon}, v_{a}^{\varepsilon}, v_{b}^{\varepsilon}, v_{0}^{\varepsilon}, \theta_{0}^{\varepsilon}\right)$ and $\left(\hat{f}^{\varepsilon}, \hat{v}_{a}^{\varepsilon}, \hat{v}_{b}^{\varepsilon}, \hat{v}_{0}^{\varepsilon}, \hat{\theta}_{0}^{\varepsilon}\right)$, and if these data satisfy (2.7)-(2.11) for the same constant $K$, their difference satisfies

$$
\left\{\begin{array}{c}
\left\|v^{\varepsilon}-\hat{v}^{\varepsilon}\right\|_{L^{\infty}\left(0 ; T ; L^{2}(\Omega)\right)}+\left\|v^{\varepsilon}-\hat{v}^{\varepsilon}\right\|_{L^{2}\left(0, T ; H^{1}(\Omega)\right)}+\left\|\theta^{\varepsilon}-\hat{\theta}^{\varepsilon}\right\|_{L^{\infty}\left(0, T ; L^{1}(\Omega)\right)} \\
\leq C^{\star \star}\left(\left\|f^{\varepsilon}-\hat{f}^{\varepsilon}\right\|_{L^{2}(Q)}+\left\|v_{a}^{\varepsilon}-\hat{v}_{a}^{\varepsilon}\right\|_{H^{1}(0, T)}+\left\|v_{b}^{\varepsilon}-\hat{v}_{b}^{\varepsilon}\right\|_{H^{1}(0, T)}\right. \\
\left.+\left\|v_{0}^{\varepsilon}-\hat{v}_{0}^{\varepsilon}\right\|_{L^{2}(\Omega)}+\left\|\theta_{0}^{\varepsilon}-\hat{\theta}_{0}^{\varepsilon}\right\|_{L^{1}(\Omega)}\right)
\end{array}\right.
$$

where $C^{\star \star}$ denotes a constant which depends only on $c_{1}, c_{2}, c_{3}, c_{4}, c_{5}, c_{6}, c_{7},(b-a)$ and $K$. 
Observe that every term in equations (2.14) and (2.15) has a meaning in the sense of distributions, since $\mu^{\varepsilon}\left(x, \theta^{\varepsilon}\right)$ belongs to $L^{\infty}(Q)$. Similarly the boundary conditions (2.16) have a meaning, since $v^{\varepsilon}$ belongs to $L^{\infty}\left(0, T ; H^{1}(\Omega)\right)$ and since $H^{1}(\Omega) \subset C^{0}(\bar{\Omega})$. Finally the initial conditions (2.17) and (2.18) have a meaning since $v^{\varepsilon}$ belongs to $H^{1}\left(0, T ; L^{2}(\Omega)\right) \subset C^{0}\left([0, T] ; L^{2}(\Omega)\right)$ and since $\theta^{\varepsilon}$ belongs to $W^{1,1}\left(0, T ; L^{1}(\Omega)\right) \subset$ $\subset C^{0}\left([0, T] ; L^{1}(\Omega)\right)$.

The regularity (2.20) of the stress $\sigma^{\varepsilon}$ is very specific to the fact that the problem is one-dimensional. This regularity immediately follows, for the first statement, from the definition (2.19) of $\sigma^{\varepsilon}$ and from (2.2), (2.12), and for the second statement, from equation (2.14), which reads as $\frac{\partial \sigma^{\varepsilon}}{\partial x}=\rho^{\varepsilon} \frac{\partial v^{\varepsilon}}{\partial t}-f^{\varepsilon}$, and from (2.5), (2.12) and (2.7).

REMARK 2.2 (Neumann and mixed boundary conditions). As pointed out in [8], a result similar to the result of Theorem 2.1 still holds true when the Dirichlet boundary conditions (1.5) on $v^{\varepsilon}$ are replaced either by the Neumann boundary conditions (1.6) on $\sigma^{\varepsilon}$ or by the mixed boundary conditions (1.7) on $v^{\varepsilon}$ and $\sigma^{\varepsilon}$.

In those cases, we consider sequences of boundary conditions $\sigma_{a}^{\varepsilon}$ and $\sigma_{b}^{\varepsilon}$ which satisfy

$$
\left\{\begin{array}{l}
\sigma_{a}^{\varepsilon} \in L^{2}(0, T), \quad \sigma_{b}^{\varepsilon} \in L^{2}(0, T), \quad \sigma_{a}^{0} \in L^{2}(0, T), \quad \sigma_{b}^{0} \in L^{2}(0, T), \\
\left\|\sigma_{a}^{\varepsilon}\right\|_{L^{2}(0, T)} \leq K, \quad\left\|\sigma_{b}^{\varepsilon}\right\|_{L^{2}(0, T)} \leq K, \\
\sigma_{a}^{\varepsilon} \rightarrow \sigma_{a}^{0} \quad \text { in } L^{2}(0, T) \text { weak, } \quad \sigma_{b}^{\varepsilon} \rightarrow \sigma_{a}^{0} \quad \text { in } L^{2}(0, T) \text { weak, }
\end{array}\right.
$$

when Neumann boundary conditions are concerned, and

$$
\left\{\begin{array}{l}
v_{a}^{\varepsilon} \in H^{1}(0, T), \quad \sigma_{b}^{\varepsilon} \in L^{2}(0, T), \quad v_{a}^{0} \in H^{1}(0, T), \quad \sigma_{b}^{0} \in L^{2}(0, T), \\
\left\|v_{a}^{\varepsilon}\right\|_{H^{1}(0, T)} \leq K, \quad\left\|\sigma_{b}^{\varepsilon}\right\|_{L^{2}(0, T)} \leq K, \\
v_{a}^{\varepsilon} \rightarrow v_{a}^{0} \quad \text { in } H^{1}(0, T) \text { weak, } \quad \sigma_{b}^{\varepsilon} \rightarrow \sigma_{a}^{0} \quad \text { in } L^{2}(0, T) \text { weak, }
\end{array}\right.
$$

with the compatibility condition

$$
v_{0}^{\varepsilon}(a)=v_{a}^{\varepsilon}(0),
$$

when mixed boundary conditions are concerned.

Under these hypotheses, results of existence, uniqueness and local Lipschitz continuity with respect to the data similar to Theorem 2.1] continue to hold true in the case of Neumann or mixed boundary conditions.

2.3. Regularity results. In this subsection, we state two regularity results on $\sigma^{\varepsilon}$ and $\frac{\partial v^{\varepsilon}}{\partial t}$ which will play a crucial role in the proof of the homogenization result.

When $\Omega=(a, b) \subset \mathbf{R}$, an interpolation result (see Lemma 3.1 of $[8]$ ) asserts that

$$
L^{\infty}\left(0, T ; L^{2}(\Omega)\right) \cap L^{2}\left(0, T ; H^{1}(\Omega)\right) \subset L^{6}(Q) ;
$$

this result is specific to the one-dimensional case. Combined with (2.20) and (2.23), this immediately implies the following regularity result on $\sigma^{\varepsilon}$. 
Proposition 2.3 ( $L^{6}$ regularity of $\sigma$ ). Assume that the hypotheses of Theorem 2.1 hold true. Then the stress $\sigma^{\varepsilon}$ defined by (2.19) satisfies

$$
\sigma^{\varepsilon} \in L^{6}(Q), \quad\left\|\sigma^{\varepsilon}\right\|_{L^{6}(Q)} \leq C C^{\star},
$$

where $C^{\star}$ is the constant which appears in the a priori estimate (2.23) and where $C$ is a constant which depends only on $(b-a)$.

On the other hand, as stated by the following theorem, $\frac{\partial v^{\varepsilon}}{\partial t}$ is bounded in $L_{l o c}^{\infty}\left(0, T ; L_{l o c}^{2}(\Omega)\right) \cap L_{l o c}^{2}\left((0, T) ; H_{l o c}^{1}(\Omega)\right)$, when, in addition to the above hypotheses, $\frac{\partial f^{\varepsilon}}{\partial t}$ is bounded in $L^{2}\left(0, T ; H^{-1}(\Omega)\right)$.

Theorem 2.4 (Regularity of $\frac{\partial v}{\partial t}$ ). Assume that the hypotheses of Theorem 2.1 hold true and that

$$
\left\{\begin{array}{l}
\frac{\partial f^{\varepsilon}}{\partial t} \in L^{2}\left(0, T ; H^{-1}(\Omega)\right), \\
\left\|\frac{\partial f^{\varepsilon}}{\partial t}\right\|_{L^{2}\left(0, T ; H^{-1}(\Omega)\right)} \leq K^{\prime} .
\end{array}\right.
$$

For $\delta>0$ sufficiently small, denote by $\Omega_{\delta}$ the interval

$$
\Omega_{\delta}=(a+\delta, b-\delta) .
$$

Then the unique solution $\left(v^{\varepsilon}, \theta^{\varepsilon}\right)$ of (2.12) $-(2.18)$ satisfies

$$
\frac{\partial v^{\varepsilon}}{\partial t} \in L^{\infty}\left(\delta, T-\delta ; L^{2}\left(\Omega_{\delta}\right)\right) \cap L^{2}\left(\delta, T-\delta ; H^{1}\left(\Omega_{\delta}\right)\right),
$$

with

$$
\left\|\frac{\partial v^{\varepsilon}}{\partial t}\right\|_{L^{\infty}\left(\delta, T ; L^{2}\left(\Omega_{\delta}\right)\right)}+\left\|\frac{\partial v^{\varepsilon}}{\partial t}\right\|_{L^{2}\left(\delta, T ; H^{1}\left(\Omega_{\delta}\right)\right)} \leq C_{\delta}^{\star},
$$

where $C_{\delta}^{\star}$ denotes a constant which depends only on $c_{1}, c_{2}, c_{3}, c_{4}, c_{5}, c_{6}, c_{7}, b-a, K$, $K^{\prime}$ and $\delta$.

Theorem 2.4 is similar to Theorem 3.2 of $[\underline{8}$, except for the fact that the regularity is now local in time (i.e. in $(\delta, T-\delta)$ and not in $(0, T))$ as well as in space (i.e. in $\Omega_{\delta}$ and not in $\Omega$ ). This theorem will be proved in Section 4 by a rather classical proof.

Similar regularity results hold true as far as Neumann and mixed conditions are concerned.

2.4. Homogenization result. The main result of the present paper is the following.

Theorem 2.5 (Homogenization). Consider sequences of viscosity functions $\mu^{\varepsilon}$, heat coefficients $c^{\varepsilon}$, densities $\rho^{\varepsilon}$, external forces $f^{\varepsilon}$, boundary conditions $v_{a}^{\varepsilon}$ and $v_{b}^{\varepsilon}$ and initial conditions $v_{0}^{\varepsilon}$ and $\theta_{0}^{\varepsilon}$, which satisfy (2.1)-(2.11). In addition to (2.7), assume that either (2.30), i.e.

or

$$
\frac{\partial f^{\varepsilon}}{\partial t} \quad \text { is bounded in } L^{2}\left(0, T ; H^{-1}(\Omega)\right)
$$

$$
f^{\varepsilon} \rightarrow f^{0} \quad \text { in } L^{2}(Q) \text { strong, }
$$

holds true. 
Then there exist a subsequence $\varepsilon^{\prime}$, a homogenized viscosity function $\mu^{0}$ and a homogenized heat coefficient $c^{0}$ which satisfy (2.1) (with a constant $c_{7}^{0}$ possibly different from $c_{7}$ ), (2.2), (2.3) (with a modulus of continuity $\omega^{0}$ possibly different from $\omega$ ) and (2.4) (with constants $c_{3}^{0}$ and $c_{4}^{0}$ possibly different from $c_{3}$ and $c_{4}$ ), such that the unique solution $\left(v^{\varepsilon}, \theta^{\varepsilon}\right)$ of (2.12)-(2.18) and the stress $\sigma^{\varepsilon}$ defined by (2.19) satisfy

$$
\begin{gathered}
v^{\varepsilon^{\prime}} \rightarrow v^{0} \quad \text { in } L^{\infty}\left(0, T ; H^{1}(\Omega)\right) \text { weak-star, } \\
\frac{\partial v^{\varepsilon^{\prime}}}{\partial t} \rightarrow \frac{\partial v^{0}}{\partial t} \quad \text { in } L^{2}\left(0, T ; L^{2}(\Omega)\right) \text { weak }, \\
\theta^{\varepsilon^{\prime}} \rightarrow \theta^{0} \quad \text { in } \mathcal{D}^{\prime}(Q) \text { weak-star, } \\
\left\{\begin{aligned}
\sigma^{\varepsilon^{\prime}} \rightarrow \sigma^{0} & \text { in } L^{\infty}\left(0, T ; L^{2}(\Omega)\right) \text { weak-star } \\
& \text { and in } L^{2}\left(0, T ; H^{1}(\Omega)\right) \cap L^{6}(Q) \text { weak },
\end{aligned}\right.
\end{gathered}
$$

where $\left(v^{0}, \theta^{0}\right)$ is the unique solution of (2.12)-(2.18) for the viscosity function $\mu^{0}$, the heat coefficient $c^{0}$, the density $\rho^{0}$, the external force $f^{0}$, the boundary conditions $v_{a}^{0}$ and $v_{b}^{0}$ and the initial conditions $v^{0}$ and $\theta^{0}$, and where the stress $\sigma^{0}$ is defined by

$$
\sigma^{0}=\mu^{0}\left(x, \theta^{0}\right) \frac{\partial v^{0}}{\partial x}
$$

Theorem 2.5 will be proved in Section 5

REMARK 2.6 (Summary of Theorem 2.5). Theorem 2.5 asserts that there exist a subsequence $\varepsilon^{\prime}$, a homogenized viscosity function $\mu_{0}(x, s)$ and a homogenized heat conduction coefficient $c_{0}(x, s)$.

Actually the subsequence $\varepsilon^{\prime}$ and the homogenized functions $\mu^{0}$ and $c^{0}$ depend only (and do depend) on the sequences $\mu^{\varepsilon}, c^{\varepsilon}$ and $\theta_{0}^{\varepsilon}$, but do not depend on the other data $\rho^{\varepsilon}, f^{\varepsilon}, v_{a}^{\varepsilon}, v_{b}^{\varepsilon}$ and $v_{0}^{\varepsilon}$.

The functions $\mu^{0}$ and $c^{0}$ are explicitly constructed through a rather complicated process, which involves weak limits (for the subsequence $\varepsilon^{\prime}$ and for every $s$ fixed) of some functions of $\mu^{\varepsilon}(x, s), c^{\varepsilon}(x, s)$ and $\theta_{0}^{\varepsilon}$. This process will be described in Section 3 below, where the functions $\mu^{0}$ and $c^{0}$ as well as the subsequence $\varepsilon^{\prime}$ will be defined (see Propositions 3.3 and 3.4 see also Remark 3.5 where this process is summarized).

REMARK 2.7 (Stability by homogenization of $c^{\varepsilon}(x, s)$ ). Theorem 2.5 proves that the system (2.12)-(2.18) is stable by homogenization, or in other terms is stable for sequences of viscosity functions $\mu^{\varepsilon}$, heat coefficients $c^{\varepsilon}$ and densities $\rho^{\varepsilon}$ which satisfy (2.1)-(2.5).

Note however that the subclass where $c^{\varepsilon}$ only depends on $x$, i.e. the class where

$$
\left\{\begin{array}{l}
c^{\varepsilon}(x, s)=c^{\varepsilon}(x) \quad \text { with } c^{\varepsilon}(x) \in L^{\infty}(\Omega), \\
c_{3} \leq c^{\varepsilon}(x) \leq c_{4} \quad \text { a.e. } x \in \Omega,
\end{array}\right.
$$

is not stable by homogenization. Indeed we will exhibit in Section 6 below a sequence of heat coefficients $c^{\varepsilon}(x)$ satisfying (2.41) for which the homogenized heat coefficient $c^{0}$ (in the sense of Theorem 2.5) is a heat coefficient $c^{0}(x, s)$ which does depend on $s$. 
REMARK 2.8 (Oscillation and nonoscillation of the unknowns). Let us examine in more detail the behaviour of the quantities $v^{\varepsilon}, \frac{\partial v^{\varepsilon}}{\partial t}, \frac{\partial v^{\varepsilon}}{\partial x}, \sigma^{\varepsilon}, \theta^{\varepsilon}$ and $\frac{\partial \theta^{\varepsilon}}{\partial t}$. We will assume in this remark that hypothesis (2.34) holds true.

In view of (2.21), the velocities $v^{\varepsilon}$ are bounded in $H^{1}(Q)$, and therefore do not oscillate. On the other hand, in view of (2.32) and of Aubin's compactness lemma, the accelerations $\frac{\partial v^{\varepsilon}}{\partial t}$ are relatively compact in $L_{l o c}^{2}(Q)$, and therefore do not oscillate. Finally in view of (2.23), the stresses $\sigma^{\varepsilon}$ are bounded in $L^{2}\left(0, T ; H^{1}(\Omega)\right)$. Also, since

$$
\left\{\begin{aligned}
\frac{\partial \sigma^{\varepsilon}}{\partial t} & =\mu^{\varepsilon}\left(x, \theta^{\varepsilon}\right) \frac{\partial^{2} v^{\varepsilon}}{\partial x \partial t}+\frac{\partial \mu^{\varepsilon}}{\partial s}\left(x, \theta^{\varepsilon}\right) \frac{\partial \theta^{\varepsilon}}{\partial t} \frac{\partial v^{\varepsilon}}{\partial x} \\
& =\mu^{\varepsilon}\left(x, \theta^{\varepsilon}\right) \frac{\partial^{2} v^{\varepsilon}}{\partial x \partial t}+\frac{\partial \mu^{\varepsilon}}{\partial s}\left(x, \theta^{\varepsilon}\right) \frac{1}{\left(\mu^{\varepsilon}\left(x, \theta^{\varepsilon}\right)\right)^{2} c^{\varepsilon}\left(x, \theta^{\varepsilon}\right)}\left(\sigma^{\varepsilon}\right)^{3},
\end{aligned}\right.
$$

the stress rates $\frac{\partial \sigma^{\varepsilon}}{\partial t}$ are bounded in $L_{l o c}^{2}(Q)+L^{2}(Q) \subset L_{l o c}^{2}(Q)$ in view of (2.32) and (2.29). Thus the stresses $\sigma^{\varepsilon}$ are bounded in $H_{l o c}^{1}(Q)$, and therefore do not oscillate.

On the other hand, in view of (2.22), the temperatures $\theta^{\varepsilon}$ are bounded in $W^{1,1}\left(0, T ; L^{1}(\Omega)\right)$, and therefore do not oscillate in time. Similarly the strain rates $\frac{\partial v^{\varepsilon}}{\partial x}$, which are given by

$$
\frac{\partial v^{\varepsilon}}{\partial x}=\frac{1}{\mu^{\varepsilon}\left(x, \theta^{\varepsilon}\right)} \sigma^{\varepsilon}
$$

do not oscillate in time since the stresses $\sigma^{\varepsilon}$ do not oscillate and since the temperatures $\theta^{\varepsilon}$ (and therefore the coefficients $\mu^{\varepsilon}\left(x, \theta^{\varepsilon}\right)$ ) do not oscillate in time. Finally the temperature increases $\frac{\partial \theta^{\varepsilon}}{\partial t}$, which are given by

$$
\frac{\partial \theta^{\varepsilon}}{\partial t}=\frac{1}{\mu^{\varepsilon}\left(x, \theta^{\varepsilon}\right) c^{\varepsilon}\left(x, \theta^{\varepsilon}\right)}\left(\sigma^{\varepsilon}\right)^{2}
$$

do not oscillate in time for the same reason.

In contrast, we claim that the temperatures $\theta^{\varepsilon}$ do oscillate in space, which implies, in view of (2.42) and (2.43), that the strain rates $\frac{\partial v^{\varepsilon}}{\partial x}$ and the temperature increases $\frac{\partial \theta^{\varepsilon}}{\partial t}$ also oscillate in space. Indeed in the proofs below (see Sections 3 , 4 and 5) (and already in the proofs in [7] and [8]), very important quantities, herein named the transformed temperatures $\tau^{\varepsilon}$ (see (3.19) below), naturally appear. These transformed temperatures $\tau^{\varepsilon}$ are proved to be bounded in $W^{1,1}(Q)$ (see (3.34) below), and therefore do not oscillate. Such is not the case for the temperatures $\theta^{\varepsilon}$, since the formula

$$
\theta^{\varepsilon}(t, x)=N^{\varepsilon}\left(x, \tau^{\varepsilon}(t, x)\right)
$$

(see (3.20) below) implies that the temperatures $\theta^{\varepsilon}$ do oscillate in space.

REMARK 2.9 (Estimates on the constants). Estimates on the constants $c_{7}^{0}, c_{3}^{0}, c_{4}^{0}$ and on the modulus of continuity $\omega^{0}$ in terms of $c_{1}, c_{2}, c_{3}, c_{4}, c_{7}$ and $\omega$ are given in (3.49), (3.50) and (3.51). In particular, when the functions $c^{\varepsilon}(x, s)$ are uniformly (in $x$ and $\varepsilon$ ) Lipschitz continuous in $s$, i.e. when $\omega(|s|)=c_{8}|s|$, then $c^{0}$ is also uniformly 
(in $x$ ) Lipschitz continuous in $s$ with a constant $c_{8}^{0}$ possibly different from $c_{8}$ (see Remark 3.7).

REMARK 2.10 (Nonequivalence of hypotheses (2.34) and (2.35)). Hypotheses (2.7) and (2.34) do not imply (2.35), and conversely hypothesis (2.35) does not imply (2.34). Therefore neither of the two hypotheses (2.34) and (2.35) reduces to the other one, even if they are similar in spirit.

REMARK 2.11 (Neumann and mixed boundary conditions). Homogenization results similar to Theorem 2.5 hold true as far as Neumann and mixed boundary conditions are concerned. We leave their statements and proofs to the reader. Let us emphasize that the homogenized viscosity function $\mu^{0}$ and heat coefficient $c^{0}$ are independent of the type of the boundary conditions and are identical to the ones which appear in Theorem 2.5 ,

\section{Definition of the homogenized viscosity function $\mu^{0}$ and of the homoge- nized heat coefficient $c^{0}$.}

3.1. Transformation of the problem. As in [7] and [8, we will perform in this subsection a change of unknown functions, and we will write the system (2.12)-(2.18) in an equivalent but simpler form. Although this transformation is not needed in order to define the functions $\mu^{0}$ and $c^{0}$, it motivates these definitions and plays an important role in the proof of Theorem 2.5. In this subsection, we will also give a regularity result on a very important quantity, the transformed temperature.

We first make a translation which reduces the problem to homogeneous boundary conditions. (Such a translation does not have to be done when Neumann boundary conditions are concerned, and it has to be conveniently modified when mixed boundary conditions are concerned.) We define

$$
\begin{gathered}
\bar{v}^{\varepsilon}(t, x)=\frac{(x-a) v_{b}^{\varepsilon}(t)+(b-x) v_{a}^{\varepsilon}(t)}{b-a}, \\
u_{0}^{\varepsilon}(x)=v_{0}^{\varepsilon}(x)-\bar{v}^{\varepsilon}(0, x), \\
u^{\varepsilon}(t, x)=v^{\varepsilon}(t, x)-\bar{v}^{\varepsilon}(t, x) .
\end{gathered}
$$

Note that

$$
u_{0}^{\varepsilon} \in H_{0}^{1}(\Omega),
$$

when $v_{0}^{\varepsilon}, v_{a}^{\varepsilon}$ and $v_{b}^{\varepsilon}$ satisfy hypotheses (2.8), (2.9) and (2.10).

Then $\left(v^{\varepsilon}, \theta^{\varepsilon}\right)$ is a solution of (2.12)-(2.18) if and only if $\left(u^{\varepsilon}, \theta^{\varepsilon}\right)$ is a solution of

$$
\begin{gathered}
\rho^{\varepsilon}(x) \frac{\partial u^{\varepsilon}}{\partial t}-\frac{\partial}{\partial x}\left(\mu^{\varepsilon}\left(x, \theta^{\varepsilon}\right)\left(\frac{\partial u^{\varepsilon}}{\partial x}+\frac{\partial \bar{v}^{\varepsilon}}{\partial x}\right)\right)=f^{\varepsilon}-\rho^{\varepsilon}(x) \frac{\partial \bar{v}^{\varepsilon}}{\partial t} \quad \text { in } \mathcal{D}^{\prime}(Q), \\
c^{\varepsilon}\left(x, \theta^{\varepsilon}\right) \frac{\partial \theta^{\varepsilon}}{\partial t}=\mu^{\varepsilon}\left(x, \theta^{\varepsilon}\right)\left(\frac{\partial u^{\varepsilon}}{\partial x}+\frac{\partial \bar{v}^{\varepsilon}}{\partial x}\right)^{2} \quad \text { in } \mathcal{D}^{\prime}(Q) \\
u^{\varepsilon}(t, a)=0, \quad u^{\varepsilon}(t, b)=0 \quad \text { a.e. } t \in(0, T) \\
u^{\varepsilon}(0, x)=u_{0}^{\varepsilon}(x) \quad \text { a.e. } x \in \Omega \\
\theta^{\varepsilon}(0, x)=\theta_{0}^{\varepsilon}(x) \quad \text { a.e. } x \in \Omega
\end{gathered}
$$


We then perform a change of unknown function by replacing $\theta(t, x)$ by a new unknown denoted by $\tau(t, x)$. We define the functions $M^{\varepsilon}: \Omega \times \mathbf{R} \rightarrow \mathbf{R}$ by

$$
M^{\varepsilon}(x, s)=\int_{\theta_{0}^{\varepsilon}(x)}^{s} c^{\varepsilon}\left(x, s^{\prime}\right) \mu^{\varepsilon}\left(x, s^{\prime}\right) d s^{\prime} \quad \text { a.e. } x \in \Omega, \forall s \in \mathbf{R} .
$$

Since in view of (2.2) and (2.4) one has

$$
0<c_{3} c_{1} \leq c^{\varepsilon}\left(x, s^{\prime}\right) \mu^{\varepsilon}\left(x, s^{\prime}\right) \leq c_{4} c_{2}<+\infty,
$$

the functions $s \rightarrow M^{\varepsilon}(x, s)$ are Carathéodory functions which are uniformly (in $x$ and $\varepsilon$ ) Lipschitz continuous in $s$ and which satisfy

$$
c_{3} c_{1} \leq \frac{\partial M^{\varepsilon}}{\partial s}(x, s) \leq c_{4} c_{2} \quad \text { a.e. } x \in \Omega, \forall s \in \mathbf{R} .
$$

Moreover the functions $s \rightarrow \frac{\partial M^{\varepsilon}}{\partial s}(x, s)=c^{\varepsilon}(x, s) \mu^{\varepsilon}(x, s)$ are Carathéodory functions which are uniformly (in $x$ and $\varepsilon$ ) continuous in $s$, since in view of (2.1)-(2.4) one has

$$
\left\{\begin{array}{r}
\left|\frac{\partial M^{\varepsilon}}{\partial s}(x, s)-\frac{\partial M^{\varepsilon}}{\partial s}\left(x, s^{\prime}\right)\right| \leq c_{7} c_{4}\left|s-s^{\prime}\right|+c_{2} \omega\left(\left|s-s^{\prime}\right|\right) \\
\text { a.e. } x \in \Omega, \quad \forall s, s^{\prime} \in \mathbf{R} .
\end{array}\right.
$$

Finally, for almost every $x \in \Omega$, the function $s \rightarrow M^{\varepsilon}(x, s)$ is one-to-one from $\mathbf{R}$ onto $\mathbf{R}$ and satisfies

$$
M^{\varepsilon}\left(x, \theta_{0}^{\varepsilon}(x)\right)=0 \quad \text { a.e. } x \in \Omega .
$$

We define $N^{\varepsilon}(x, r)$ as the reciprocal function of $M^{\varepsilon}(x, s)$, i.e.

$$
M^{\varepsilon}(x, s)=r \quad \Longleftrightarrow \quad N^{\varepsilon}(x, r)=s \quad \text { a.e. } x \in \Omega, \quad \forall s, r \in \mathbf{R} .
$$

The function $r \rightarrow N^{\varepsilon}(x, r)$ is a one-to-one function from $\mathbf{R}$ onto $\mathbf{R}$ and satisfies

$$
N^{\varepsilon}(x, 0)=\theta_{0}^{\varepsilon}(x) \quad \text { a.e. } x \in \Omega \text {. }
$$

Since $M^{\varepsilon}\left(x, N^{\varepsilon}(x, r)\right)=r$, the chain rule yields

$$
\frac{\partial M^{\varepsilon}}{\partial s}\left(x, N^{\varepsilon}(x, r)\right) \frac{\partial N^{\varepsilon}}{\partial r}(x, r)=1 .
$$

Thus the functions $r \rightarrow N^{\varepsilon}(x, r)$ are Carathéodory functions which are uniformly (in $x$ and $\varepsilon$ ) Lipschitz continuous in $s$ and which satisfy

$$
\frac{1}{c_{4} c_{2}} \leq \frac{\partial N^{\varepsilon}}{\partial r}(x, r) \leq \frac{1}{c_{3} c_{1}} \quad \text { a.e. } x \in \Omega, \forall s \in \mathbf{R} .
$$

Moreover, the functions $s \rightarrow \frac{\partial N^{\varepsilon}}{\partial r}(x, r)$ are Carathéodory functions which are uniformly (in $x$ and $\varepsilon$ ) continuous in $s$, since in view of (3.16), (3.11) and (3.12), one has

$$
\left\{\begin{array}{l}
\left|\frac{\partial N^{\varepsilon}}{\partial r}(x, r)-\frac{\partial N^{\varepsilon}}{\partial r}\left(x, r^{\prime}\right)\right|=\frac{\left|\frac{\partial M^{\varepsilon}}{\partial s}\left(x, N^{\varepsilon}(x, r)\right)-\frac{\partial M^{\varepsilon}}{\partial s}\left(x, N^{\varepsilon}\left(x, r^{\prime}\right)\right)\right|}{\frac{\partial M^{\varepsilon}}{\partial s}\left(x, N^{\varepsilon}(x, r)\right) \frac{\partial M^{\varepsilon}}{\partial s}\left(x, N^{\varepsilon}\left(x, r^{\prime}\right)\right)} \\
\leq \frac{1}{\left(c_{3} c_{1}\right)^{2}}\left(c_{7} c_{4}\left|N^{\varepsilon}(x, r)-N^{\varepsilon}\left(x, r^{\prime}\right)\right|+c_{2} \omega\left(\left|N^{\varepsilon}(x, r)-N^{\varepsilon}\left(x, r^{\prime}\right)\right|\right)\right),
\end{array}\right.
$$


which using (3.17) implies

$$
\left\{\begin{array}{r}
\left|\frac{\partial N^{\varepsilon}}{\partial r}(x, r)-\frac{\partial N^{\varepsilon}}{\partial r}\left(x, r^{\prime}\right)\right| \leq \frac{c_{7} c_{4}}{c_{3}^{3} c_{1}^{3}}\left|r-r^{\prime}\right|+\frac{c_{2}}{c_{3}^{2} c_{1}^{2}} \omega\left(\frac{\left|r-r^{\prime}\right|}{c_{3} c_{1}}\right) \\
\text { a.e. } x \in \Omega, \quad \forall r, r^{\prime} \in \mathbf{R} .
\end{array}\right.
$$

We now define the transformed temperature $\tau^{\varepsilon}$ by

$$
\tau^{\varepsilon}(t, x)=M^{\varepsilon}\left(x, \theta^{\varepsilon}(t, x)\right) .
$$

In view of the definition (3.14) of the reciprocal function $N^{\varepsilon}$, definition (3.19) is equivalent to

$$
\theta^{\varepsilon}(t, x)=N^{\varepsilon}\left(x, \tau^{\varepsilon}(t, x)\right) .
$$

The reason for the definition of the transformed temperature $\tau^{\varepsilon}$ is that, multiplying equation (3.6) by $\mu^{\varepsilon}\left(x, \theta^{\varepsilon}\right)$ (which is bounded from above and from below by strictly positive constants), we see that (3.6) is equivalent to

$$
\frac{\partial \tau^{\varepsilon}}{\partial t}=\left|\mu^{\varepsilon}\left(x, \theta^{\varepsilon}\right)\left(\frac{\partial u^{\varepsilon}}{\partial x}+\frac{\partial \bar{v}^{\varepsilon}}{\partial x}\right)\right|^{2} \quad \text { in } \mathcal{D}^{\prime}(Q),
$$

while the initial condition (3.9) is equivalent to

$$
\tau^{\varepsilon}(0, x)=0 \quad \text { a.e. } x \in \Omega .
$$

We finally define the function $\lambda^{\varepsilon}: \Omega \times \mathbf{R} \rightarrow \mathbf{R}$ by

$$
\lambda^{\varepsilon}(x, r)=\mu^{\varepsilon}\left(x, N^{\varepsilon}(x, r)\right) \quad \text { a.e. } x \in \Omega, \forall r \in \mathbf{R} .
$$

In view of (2.1), (2.2) and (3.17) the functions $\lambda^{\varepsilon}$ are Carathéodory functions which are Lipschitz continuous in $r$ and which satisfy

$$
\begin{gathered}
c_{1} \leq \lambda^{\varepsilon}(x, r) \leq c_{2} \quad \text { a.e. } x \in \Omega, \forall r \in \mathbf{R}, \\
-\frac{c_{7}}{c_{3} c_{1}} \leq \frac{\partial \lambda^{\varepsilon}}{\partial r}(x, r) \leq 0 \quad \text { a.e. } x \in \Omega, \forall r \in \mathbf{R} .
\end{gathered}
$$

Also one has

$$
\begin{gathered}
\mu^{\varepsilon}\left(x, \theta^{\varepsilon}(t, x)\right)=\lambda^{\varepsilon}\left(x, \tau^{\varepsilon}(t, x)\right) \\
\sigma^{\varepsilon}(t, x)=\mu^{\varepsilon}\left(x, \theta^{\varepsilon}(t, x)\right) \frac{\partial v^{\varepsilon}}{\partial x}(t, x)=\lambda^{\varepsilon}\left(x, \tau^{\varepsilon}(t, x)\right)\left(\frac{\partial u^{\varepsilon}}{\partial x}(t, x)+\frac{\partial \bar{v}^{\varepsilon}}{\partial x}(t, x)\right) .
\end{gathered}
$$

It is now straightforward to prove the following equivalence result.

Proposition 3.1 (Equivalence). Assume that hypotheses (2.1)-2.11) hold true, and define $\bar{v}^{\varepsilon}, u_{0}^{\varepsilon}, M^{\varepsilon}, N^{\varepsilon}$ and $\lambda^{\varepsilon}$ by (3.1), (3.2), (3.10), (3.14) and (3.21). Defining $u^{\varepsilon}$ and $\tau^{\varepsilon}$ by (3.3) and (3.19), the couple $\left(v^{\varepsilon}, \theta^{\varepsilon}\right)$ satisfies (2.12)-(2.18) if and only if the 
couple $\left(u^{\varepsilon}, \tau^{\varepsilon}\right)$ satisfies

$$
\begin{aligned}
& u^{\varepsilon} \in L^{\infty}\left(0, T ; H_{0}^{1}(\Omega)\right) \cap H^{1}\left(0, T ; L^{2}(\Omega)\right), \\
& \tau^{\varepsilon} \in W^{1,1}\left(0, T ; L^{1}(\Omega)\right), \\
& \rho^{\varepsilon}(x) \frac{\partial u^{\varepsilon}}{\partial t}-\frac{\partial}{\partial x}\left(\lambda^{\varepsilon}\left(x, \tau^{\varepsilon}\right)\left(\frac{\partial u^{\varepsilon}}{\partial x}+\frac{\partial \bar{v}^{\varepsilon}}{\partial x}\right)\right)=f^{\varepsilon}-\rho^{\varepsilon}(x) \frac{\partial \bar{v}^{\varepsilon}}{\partial t} \text { in } \mathcal{D}^{\prime}(Q), \\
& \frac{\partial \tau^{\varepsilon}}{\partial t}=\left|\lambda^{\varepsilon}\left(x, \tau^{\varepsilon}\right)\left(\frac{\partial u^{\varepsilon}}{\partial x}+\frac{\partial \bar{v}^{\varepsilon}}{\partial x}\right)\right|^{2} \quad \text { in } \mathcal{D}^{\prime}(Q), \\
& u^{\varepsilon}(0, x)=u_{0}^{\varepsilon}(x) \quad \text { a.e. } x \in \Omega, \\
& \tau^{\varepsilon}(0, x)=0 \quad \text { a.e. } x \in \Omega \text {. }
\end{aligned}
$$

Moreover the stress $\sigma^{\varepsilon}$ defined by (2.19) is equivalently defined by (3.25), and satisfies

$$
\sigma^{\varepsilon} \in L^{\infty}\left(0, T ; L^{2}(\Omega)\right) \cap L^{2}\left(0, T ; H^{1}(\Omega)\right) .
$$

Finally the norms of $v^{\varepsilon}$ and $\theta^{\varepsilon}$ in the spaces $L^{\infty}\left(0, T ; H^{1}(\Omega)\right) \cap$ $\cap H^{1}\left(0, T ; L^{2}(\Omega)\right)$ and $W^{1,1}\left(0, T ; L^{1}(\Omega)\right)$ and the norms of $u^{\varepsilon}$ and $\tau^{\varepsilon}$ in the spaces $L^{\infty}\left(0, T ; H_{0}^{1}(\Omega)\right) \cap H^{1}\left(0, T ; L^{2}(\Omega)\right)$ and $W^{1,1}\left(0, T ; L^{1}(\Omega)\right)$ are equivalent, with constants in the equivalences which depend only on $c_{1}, c_{2}, c_{3}, c_{4},(b-a)$ and $K$.

Note that the initial conditions (3.30) and (3.31) have a meaning since $H^{1}\left(0, T ; L^{2}(\Omega)\right) \subset C^{0}\left([0, T] ; L^{2}(\Omega)\right)$ and since $W^{1,1}\left(0, T ; L^{1}(\Omega)\right) \subset C^{0}\left([0, T] ; L^{1}(\Omega)\right)$.

Actually, the transformed temperature $\tau^{\varepsilon}$ (which belongs to $W^{1,1}\left(0, T ; L^{1}(\Omega)\right.$ ) as stated in (3.27) ) also enjoys regularity properties which are not shared by the temperature $\theta^{\varepsilon}$. Indeed we have the following result of regularity of $\tau^{\varepsilon}$ (which goes back to Proposition 3.1 of [7]; see also Proposition 3.1 of [8]).

Proposition 3.2 (Regularity of the transformed temperature). Assume that hypotheses (2.1) - (2.11) hold true. Then the transformed temperature $\tau^{\varepsilon}$ defined by (3.19) satisfies

$$
\tau^{\varepsilon} \in W^{1, \infty}\left(0, T ; L^{1}(\Omega)\right) \cap W^{1,1}\left(0, T ; H^{1}(\Omega)\right) \cap H^{1}\left(0, T ; W^{1,1}(\Omega)\right),
$$

and the following a priori estimates hold true

$$
\left\|\tau^{\varepsilon}\right\|_{W^{1, \infty}\left(0, T ; L^{1}(\Omega)\right)}+\left\|\tau^{\varepsilon}\right\|_{W^{1,1}\left(0, T ; H^{1}(\Omega)\right)}+\left\|\tau^{\varepsilon}\right\|_{H^{1}\left(0, T ; W^{1,1}(\Omega)\right)} \leq F^{\star},
$$

where $F^{\star}$ depends only on $c_{1}, c_{2}, c_{3}, c_{4}, c_{5}, c_{6}$ and $K$.

The proof of Proposition 3.2 just consists to write as (see (3.25), (3.29) and (3.31))

$$
\frac{\partial \tau^{\varepsilon}}{\partial t}=\left|\sigma^{\varepsilon}\right|^{2}, \quad \tau^{\varepsilon}(0, x)=0
$$

which using (2.20) and (2.23) implies that $\tau^{\varepsilon} \in W^{1, \infty}\left(0, T ; L^{1}(\Omega)\right)$ and the corresponding a priori estimate, but which also implies that

$$
\frac{\partial}{\partial t}\left(\frac{\partial \tau^{\varepsilon}}{\partial x}\right)=2 \sigma^{\varepsilon} \frac{\partial \sigma^{\varepsilon}}{\partial x}, \quad \frac{\partial \tau^{\varepsilon}}{\partial x}(0, x)=0
$$

which using $H^{1}(\Omega) \subset L^{\infty}(\Omega),(2.20)$ and (2.23) implies the other assertions of Proposition 3.2 
3.2. Definition of the homogenized functions $\mu^{0}$ and $c^{0}$. The goal of this subsection is to state results which define the functions $\mu^{0}$ and $c^{0}$ which appear in Theorem 2.5. These results will be proved in Subsection 3.3. For convenience the statements are divided into two propositions.

Proposition 3.3 (Definition of the subsequence $\varepsilon^{\prime}$ and of the functions $N^{0}$ and $\lambda^{0}$ ). There exist a subsequence $\varepsilon^{\prime}$ and two Carathéodory functions $N^{0}$ and $\lambda^{0}: \Omega \times \mathbf{R} \rightarrow \mathbf{R}$, with $r \rightarrow N^{0}(x, r)$ one-to-one and strictly increasing from $\mathbf{R}$ onto $\mathbf{R}$ for almost every $x \in \Omega$, which satisfy for almost every $x \in \Omega$ and for every $r, r^{\prime} \in \mathbf{R}$

$$
\begin{gathered}
N^{0}(x, 0)=\theta_{0}^{0}(x), \\
\frac{1}{c_{4} c_{2}} \leq \frac{\partial N^{0}}{\partial r}(x, r) \leq \frac{1}{c_{3} c_{1}}, \\
\left|\frac{\partial N^{0}}{\partial r}(x, r)-\frac{\partial N^{0}}{\partial r}\left(x, r^{\prime}\right)\right| \leq \frac{c_{7} c_{4}}{c_{3}^{3} c_{1}^{3}}\left|r-r^{\prime}\right|+\frac{c_{2}}{c_{3}^{2} c_{1}^{2}} \omega\left(\frac{\left|r-r^{\prime}\right|}{c_{3} c_{1}}\right), \\
c_{1} \leq \lambda^{0}(x, r) \leq c_{2}, \\
-\frac{c_{7} c_{2}^{2}}{c_{3} c_{1}^{3}} \leq \frac{\partial \lambda^{0}}{\partial r}(x, r) \leq 0,
\end{gathered}
$$

such that the functions $N^{\varepsilon}$ and $\lambda^{\varepsilon}$ defined by (3.14), (3.10) and (3.21) satisfy for every $r \in \mathbf{R}$

$$
\begin{aligned}
& N^{\varepsilon^{\prime}}(\cdot, r) \rightarrow N^{0}(\cdot, r) \quad \text { in } \mathcal{D}^{\prime}(\Omega) \text { weak-star, } \\
& \frac{1}{\lambda^{\varepsilon^{\prime}}(\cdot, r)} \rightarrow \frac{1}{\lambda^{0}(\cdot, r)} \quad \text { in } L^{\infty}(\Omega) \text { weak-star. }
\end{aligned}
$$

From $N^{0}$ and $\lambda^{0}$ defined in Proposition 3.3 , we define $\mu^{0}$ and $c^{0}$ in the following way.

Proposition 3.4 (Definition of the homogenized functions $\mu^{0}$ and $c^{0}$ ). First define $M^{0}(x, s)$ as the reciprocal function of $N^{0}(x, r)$, i.e.

$$
M^{0}(x, s)=r \quad \Longleftrightarrow \quad N^{0}(x, r)=s \quad \text { a.e. } x \in \Omega, \forall s, r \in \mathbf{R} .
$$

Then $M^{0}: \Omega \times \mathbf{R} \rightarrow \mathbf{R}$ is a Carathéodory function, with $s \rightarrow M^{0}(x, s)$ one-to-one and strictly increasing from $\mathbf{R}$ onto $\mathbf{R}$ for almost every $x \in \Omega$, which satisfies for almost every $x \in \Omega$ and for every $s, s^{\prime} \in \mathbf{R}$

$$
\begin{gathered}
M^{0}\left(x, \theta^{0}(x)\right)=0 \\
c_{3} c_{1} \leq \frac{\partial M^{0}}{\partial s}(x, s) \leq c_{4} c_{2}, \\
\left|\frac{\partial M^{0}}{\partial s}(x, s)-\frac{\partial M^{0}}{\partial s}\left(x, s^{\prime}\right)\right| \leq \frac{c_{7} c_{4}^{4} c_{2}^{3}}{c_{3}^{3} c_{1}^{3}}\left|s-s^{\prime}\right|+\frac{c_{4}^{2} c_{2}^{3}}{c_{3}^{2} c_{1}^{2}} \omega\left(\frac{c_{4} c_{2}}{c_{3} c_{1}}\left|s-s^{\prime}\right|\right) .
\end{gathered}
$$

Then define $\mu^{0}$ and $c^{0}: \Omega \times \mathbf{R} \rightarrow \mathbf{R}$ by

$$
\begin{gathered}
\mu^{0}(x, s)=\lambda^{0}\left(x, M^{0}(x, s)\right), \\
c^{0}(x, s)=\frac{\partial M^{0}}{\partial s}(x, s) \frac{1}{\mu^{0}(x, s)},
\end{gathered}
$$


for almost every $x \in \Omega$ and for every $s \in \mathbf{R}$. Then $\mu^{0}$ and $c^{0}$ are Carathéodory functions which satisfy for almost every $x \in \Omega$ and for every $s, s^{\prime} \in \mathbf{R}, s \geq s^{\prime}$

$$
\begin{gathered}
c_{1} \leq \mu^{0}(x, s) \leq c_{2}, \\
-\frac{c_{7} c_{4} c_{2}^{3}}{c_{3} c_{1}^{3}}\left(s-s^{\prime}\right) \leq \mu^{0}(x, s)-\mu^{0}\left(x, s^{\prime}\right) \leq 0, \\
\frac{c_{3} c_{1}}{c_{2}} \leq c^{0}(x, s) \leq \frac{c_{4} c_{2}}{c_{1}}, \\
\left.\mid c^{0}(x, s)-c^{0}\left(x, s^{\prime}\right)\right] \leq\left(\frac{c_{7} c_{4}^{4} c_{2}^{3}}{c_{3}^{3} c_{1}^{4}}+\frac{c_{7} c_{4}^{2} c_{2}^{4}}{c_{3} c_{1}^{5}}\right)\left|s-s^{\prime}\right|+\frac{c_{4}^{2} c_{2}^{3}}{c_{3}^{2} c_{1}^{3}} \omega\left(\frac{c_{4} c_{2}}{c_{3} c_{1}}\left|s-s^{\prime}\right|\right) .
\end{gathered}
$$

For these functions $\mu^{0}$ and $c^{0}$ and for the subsequence $\varepsilon^{\prime}$ defined in Proposition 3.3, Theorem 2.5 holds true.

REMARK 3.5 (Summary of the definitions of the homogenized functions $\mu^{0}$ and $c^{0}$ ). Let us summarize the way in which the homogenized functions $\mu^{0}$ and $c^{0}$ and the subsequence $\varepsilon^{\prime}$ are defined.

From the functions $\mu^{\varepsilon}(x, s)$ and $c^{\varepsilon}(x, s)$ and from the initial datum $\theta_{0}^{\varepsilon}(x)$, we define the function $M^{\varepsilon}(x, s)$ by (3.10), i.e. by

$$
\frac{\partial M^{\varepsilon}}{\partial s}(x, s)=c^{\varepsilon}(x, s) \mu^{\varepsilon}(x, s), \quad M^{\varepsilon}\left(x, \theta_{0}^{\varepsilon}(x)\right)=0,
$$

and the function $r \rightarrow N^{\varepsilon}(x, r)$ as the reciprocal function of $s \rightarrow M^{\varepsilon}(x, s)$ (see (3.14)). We then set (see (3.21) $)$

$$
\lambda^{\varepsilon}(x, r)=\mu^{\varepsilon}\left(x, N^{\varepsilon}(x, r)\right) .
$$

The subsequence $\varepsilon^{\prime}$ and the functions $N^{0}(x, r)$ and $\lambda^{0}(x, r)$ are then chosen such that for every fixed $r \in \mathbf{R}$ (see (3.40) and (3.41))

$$
\begin{array}{ll}
N^{\varepsilon^{\prime}}(x, r) \rightarrow N^{0}(x, r) & \text { in } \mathcal{D}^{\prime}(\Omega) \text { weak-star, } \\
\frac{1}{\lambda^{\varepsilon^{\prime}}(x, r)} \rightarrow \frac{1}{\lambda^{0}(x, r)} & \text { in } L^{\infty}(\Omega) \text { weak-star. }
\end{array}
$$

Finally, the function $s \rightarrow M^{0}(x, r)$ is defined as the reciprocal function of $r \rightarrow N^{0}(x, r)$ (see (3.42) ). We then define $\mu^{0}$ by (see (3.46))

$$
\mu^{0}(x, s)=\lambda^{0}\left(x, M^{0}(x, s)\right),
$$

and finally define $c^{0}$ by (see (3.47))

$$
c^{0}(x, s)=\frac{\partial M^{0}}{\partial s}(x, s) \frac{1}{\mu^{0}(x, s)} .
$$

In Section 66 we will give explicit formulas for $\mu^{0}$ and $c^{0}$ (see formulas (6.12) and (6.15) and Theorem 6.1) in the case of a material made of layers of given homogeneous phases characterized by densities $\rho_{i}(x)=\rho_{i}$, viscosity functions $\mu_{i}(x, s)=\mu_{i}(s)$ and heat coefficients $c_{i}(x, s)=c_{i}(s)$. 
REMARK 3.6 (Dependance of the functions $\mu^{0}$ and $c^{0}$ on $\mu^{\varepsilon}, c^{\varepsilon}$ and $\theta_{0}^{\varepsilon}$ ). Note that the subsequence $\varepsilon^{\prime}$, the homogenized viscosity function $\mu^{0}$ and the homogenized heat coefficient $c^{0}$ depend on (and depend only on) the sequences $\mu^{\varepsilon}(x, s), c^{\varepsilon}(x, s)$ and $\theta_{0}^{\varepsilon}(x)$. Let us emphasize that $\mu^{0}$ and $c^{0}$ depend on the sequence of initial temperatures $\theta_{0}^{\varepsilon}$.

REMARK 3.7 (Case where the functions $c^{\varepsilon}$ are Lipschitz continuous in $s$ ). When the functions $c^{\varepsilon}(x, s)$ are uniformly (in $x$ and $\varepsilon$ ) Lipschitz continuous in $s$, i.e. when

$$
\omega(|s|)=c_{8}|s|,
$$

or in other terms when hypothesis (2.3) reads as

$$
\left|c^{\varepsilon}(x, s)-c^{\varepsilon}\left(x, s^{\prime}\right)\right| \leq c_{8}\left|s-s^{\prime}\right| \quad \text { a.e. } x \in \Omega, \forall s, s^{\prime} \in \mathbf{R},
$$

then formula (3.51) implies that $c^{0}$ is also uniformly (in $x$ ) Lipschitz continuous in $s$ with a constant $c_{8}^{0}$ possibly different from $c_{8}$.

3.3. Proofs of Propositions 3.3 and 3.4. We begin with a classical lemma which is a basic tool in the proofs of Propositions 3.3 and 3.4

LEMma 3.8 (Extracting a weak converging subsequence for Carathéodory functions). Let $E$ be an open bounded set, $E \subset \mathbf{R}^{m}, m \geq 1$, and let $F^{\varepsilon}: E \times \mathbf{R}^{k} \rightarrow \mathbf{R}, k \geq 1$, be a sequence of Carathéodory functions which satisfy, for almost every $x \in E$ and for every $r, r^{\prime} \in \mathbf{R}^{k}$

$$
\begin{gathered}
\left|F^{\varepsilon}(x, r)-F^{\varepsilon}\left(x, r^{\prime}\right)\right| \leq \omega\left(\left|r-r^{\prime}\right|\right), \\
\left|F^{\varepsilon}(x, 0)\right| \leq G^{\varepsilon}(x),
\end{gathered}
$$

where $\omega$ is a modulus of continuity and where $G^{\varepsilon}$ is a sequence of functions such that

$$
\left\{\begin{array}{l}
G^{\varepsilon} \in L^{1}(E), G^{\varepsilon} \text { bounded in } L^{1}(E), G^{0} \in L^{1}(E), \\
G^{\varepsilon} \rightarrow G^{0} \text { in } \mathcal{D}^{\prime}(E) \text { weak-star. }
\end{array}\right.
$$

Then there exist a subsequence $\varepsilon^{\prime}$ and a Carathéodory function $F^{0}: E \times \mathbf{R}^{k} \rightarrow \mathbf{R}$ which satisfies (3.52) and

$$
\left|F^{0}(x, 0)\right| \leq G^{0}(x) \quad \text { a.e. } x \in E,
$$

such that for every fixed $r \in \mathbf{R}^{k}$,

$$
F^{\varepsilon^{\prime}}(\cdot, r) \rightarrow F^{0}(\cdot, r) \text { in } \mathcal{D}^{\prime}(E) \text { weak-star. }
$$

Moreover, if in (3.52) one has

$$
\omega(|r|)=C|r|
$$

for some constant $C>0$, i.e. if $F^{\varepsilon}$ is uniformly (in $x$ and $\varepsilon$ ) Lipschitz continuous in $r$, and if $z^{\varepsilon}$ is a sequence such that

$$
z^{\varepsilon} \rightarrow z^{0} \quad \text { in } L^{1}(E)^{k} \text { strong, }
$$

then

$$
F^{\varepsilon^{\prime}}\left(\cdot, z^{\varepsilon^{\prime}}\right) \rightarrow F^{0}\left(\cdot, z^{0}\right) \text { in } \mathcal{D}^{\prime}(E) \text { weak-star. }
$$


Proof of Lemma 3.8. First step: Definition of the subsequence $\varepsilon^{\prime}$ and of the function $F^{0}$. The estimate

$$
\left|F^{\varepsilon}(x, r)\right| \leq G_{0}^{\varepsilon}(x)+\omega(|r|) \quad \text { a.e. } x \in \Omega, \forall r \in \mathbf{R}^{k},
$$

which follows from (3.52) and (3.53), implies that $F^{\varepsilon}(\cdot, r)$ is bounded in $L^{1}(E)$ for every fixed $r \in \mathbf{R}^{k}$. Therefore one can extract a (diagonal) subsequence $\varepsilon^{\prime}$ such that for every $q \in \mathbf{Q}^{k}$

$$
F^{\varepsilon^{\prime}}(\cdot, q) \rightarrow F_{q}^{0} \quad \text { in } \mathcal{D}^{\prime}(E) \text { weak-star }
$$

where $F_{q}^{0}$ belongs to $\mathcal{D}^{\prime}(E)$.

On the other hand, for $r=0$, one deduces from (3.53), (3.54) and (3.61) that

$$
-G^{0} \leq F_{0}^{0} \leq G^{0} \quad \text { in } \mathcal{D}^{\prime}(E),
$$

which implies that $F_{0}^{0} \in L^{1}(\Omega)$ and satisfies

$$
\left|F_{0}^{0}(x)\right| \leq G^{0}(x) \quad \text { a.e. } x \in E .
$$

For every $\varphi \in C_{c}^{\infty}(E)$ one has (see (3.52) ) for every $q, q^{\prime} \in \mathbf{Q}^{k}$

$$
\left|\int_{\Omega}\left(F^{\varepsilon}(x, q)-F^{\varepsilon}\left(x^{\prime}, q^{\prime}\right)\right) \varphi(x) d x\right| \leq\|\varphi\|_{L^{1}(E)} \omega\left(\left|q-q^{\prime}\right|\right) .
$$

Passing to the limit along the subsequence $\varepsilon^{\prime}$ thanks to (3.61) implies that for every $\varphi \in C_{c}^{\infty}(E)$ and for every $q, q^{\prime} \in \mathbf{Q}^{k}$

$$
\left|\left\langle F_{q}^{0}-F_{q^{\prime}}^{0}, \varphi\right\rangle\right| \leq\|\varphi\|_{L^{1}(E)} \omega\left(\left|q-q^{\prime}\right|\right),
$$

which implies that $F_{q}^{0}-F_{q^{\prime}}^{0}$ belongs to $L^{\infty}(E)$ with

$$
\left\|F_{q}^{0}-F_{q^{\prime}}^{0}\right\|_{L^{\infty}(E)} \leq \omega\left(\left|q-q^{\prime}\right|\right) \quad \forall q, q^{\prime} \in \mathbf{Q}^{k} .
$$

Combining (3.63) and (3.62) implies that

$$
F_{q}^{0} \in L^{1}(\Omega), \quad\left\|F_{q}^{0}-F_{q^{\prime}}^{0}\right\|_{L^{1}(E)} \leq|E| \omega\left(\left|q-q^{\prime}\right|\right) \quad \forall q, q^{\prime} \in \mathbf{Q}^{k} .
$$

Therefore $F_{q_{n}}^{0}$ is a Cauchy sequence in $L^{1}(\Omega)$ if $q_{n}$ is a Cauchy sequence in $\mathbf{R}^{k}$. For every $r \in \mathbf{R}^{k}$, we define the function $F_{r}^{0}$ as the limit in $L^{1}(E)$ of any sequence $F_{q_{n}}^{0}$, where $q_{n} \in \mathbf{Q}^{k}$ is any sequence which tends to $r$ in $\mathbf{R}^{k}$. This definition is licit since $F_{r}^{0}$ depends only on $r$ and not on the sequence $q_{n}$, as is easily seen by considering two sequences $q_{n}$ and $q_{n}^{\prime}$ which tend to the same $r$.

Moreover inequality (3.63) and the definition of $F_{r}^{0}$ implies that

$$
\left\|F_{r}^{0}-F_{r^{\prime}}^{0}\right\|_{L^{\infty}(E)} \leq \omega\left(\left|r-r^{\prime}\right|\right) \quad \forall r, r^{\prime} \in \mathbf{R}^{k} .
$$

We now define the function $F^{0}: E \times \mathbf{R}^{k} \rightarrow \mathbf{R}$ by

$$
F^{0}(x, r)=F_{r}^{0}(x) \quad \text { a.e. } x \in E, \forall r \in \mathbf{R}^{k} .
$$

It immediately follows from this definition and from (3.65) and (3.62) that $F^{0}$ is a Carathéodory function which satisfies (3.52) and (3.55).

Second step: Proof of (3.56). Let us now prove that (3.56) holds true for every $r \in \mathbf{R}^{k}$ with the above-defined function $F^{0}(\cdot, r)$ and the same subsequence $\varepsilon^{\prime}$. Let $r \in \mathbf{R}^{k}$ be 
fixed. In view of (3.60) we can extract from the subsequence $\varepsilon^{\prime}$ a further subsequence, say $\varepsilon^{\prime \prime}$, which depends on $r$, such that

$$
F^{\varepsilon^{\prime \prime}}(\cdot, r) \rightarrow F_{r}^{\star} \quad \text { in } \mathcal{D}^{\prime}(E) \text { weak-star, }
$$

where $F_{r}^{\star}$ belongs to $\mathcal{D}^{\prime}(E)$. From (3.52) applied to $r$ and $r^{\prime}=q \in \mathbf{Q}^{k}$, we deduce that for every $\varphi \in C_{c}^{\infty}(\Omega)$ and every $q \in \mathbf{Q}^{k}$ one has

$$
\left|\left\langle F_{r}^{\star}-F_{q}^{0}, \varphi\right\rangle\right| \leq\|\varphi\|_{L^{1}(E)} \omega(|r-q|) .
$$

Using a sequence $q_{n} \in \mathbf{Q}^{k}$ which converges to $r$ and the definition of $F_{r}^{0}$, this implies that $F_{r}^{\star}=F_{r}^{0}$. Since the limit of the subsequence $F^{\varepsilon^{\prime \prime}}(\cdot, r)$ is uniquely determined independently of the subsequence $\varepsilon^{\prime \prime}$, the whole sequence $F^{\varepsilon^{\prime}}(\cdot, r)$ converges to $F_{r}^{0}$, which is nothing but $F^{0}(\cdot, r)$, and (3.56) is proved.

Third step: Proof of (3.59). It remains to prove that (3.59) holds true when one assumes (3.57) and (3.58). Since $z \in L^{1}(E)^{k}$, for every $\delta>0$ there exists a step function $\zeta$ (i.e. a function of the form

$$
\zeta(x)=\sum_{i} c_{i} \chi_{i}(x) \quad \text { a.e. } x \in E,
$$

where the sum is finite, where the $c_{i}$ belong to $\mathbf{R}^{k}$, and where the $\chi_{i}$ are characteristic functions of disjoints measurable sets such that $\sum_{i} \chi_{i}(x)=1$ in $E$ ), such that

$$
\left\|z^{0}-\zeta\right\|_{L^{1}(E)^{k}} \leq \delta
$$

From the formula

$$
F^{\varepsilon}(x, \zeta(x))=\sum_{i} F^{\varepsilon}\left(x, c_{i}\right) \chi_{i}(x),
$$

and from (3.56) one deduces that

$$
F^{\varepsilon^{\prime}}(\cdot, \zeta) \rightarrow F^{0}(\cdot, \zeta) \text { in } \mathcal{D}^{\prime}(E) \text { weak-star. }
$$

On the other hand, one deduces from (3.52) and (3.57) that

$$
\left\|F^{\varepsilon}\left(\cdot, z^{\varepsilon}\right)-F^{\varepsilon}(\cdot, \zeta)\right\|_{L^{1}(E)} \leq C\left\|z^{\varepsilon}-\zeta\right\|_{L^{1}(E)^{k}} .
$$

Similarly, since $F^{0}$ satisfies (3.52), one has

$$
\left\|F^{0}(\cdot, \zeta)-F^{0}\left(\cdot, z^{0}\right)\right\|_{L^{1}(E)} \leq C\left\|z^{0}-\zeta\right\|_{L^{1}(E)^{k}} \leq C \delta .
$$

The convergence (3.59) then follows from the previous results, from the convergence

$$
\left\|z^{\varepsilon}-\zeta\right\|_{L^{1}(E)^{k}} \rightarrow\left\|z^{0}-\zeta\right\|_{L^{1}(E)^{k}} \leq \delta
$$

and from the formula

$$
F^{\varepsilon^{\prime}}\left(\cdot, z^{\varepsilon^{\prime}}\right)-F^{0}\left(\cdot, z^{0}\right)=F^{\varepsilon^{\prime}}\left(\cdot, z^{\varepsilon^{\prime}}\right)-F^{\varepsilon^{\prime}}(\cdot, \zeta)+F^{\varepsilon^{\prime}}(\cdot, \zeta)-F^{0}(\cdot, \zeta)+F^{0}(\cdot, \zeta)-F^{0}\left(\cdot, z^{0}\right) .
$$

Lemma 3.8 is proved. 
Proof of Proposition 3.3. Since $N^{\varepsilon}$ satisfies (3.17) and (3.15) one has, for almost every $x \in \Omega$ and for every $r, r^{\prime} \in \mathbf{R}$

$$
\left\{\begin{array}{l}
\left|N^{\varepsilon}(x, r)-N^{\varepsilon}\left(x, r^{\prime}\right)\right| \leq \frac{1}{c_{3} c_{1}}\left|r-r^{\prime}\right|, \\
N^{\varepsilon}(x, 0)=\theta_{0}^{\varepsilon}(x), \\
\left|N^{\varepsilon}(x, r)\right| \leq \frac{1}{c_{3} c_{1}}|r|+\left|\theta_{0}^{\varepsilon}(x)\right| .
\end{array}\right.
$$

Therefore one can apply Lemma 3.8 to the sequence $N^{\varepsilon}$ (here we use the fact that $\gamma^{0} \in L^{1}(\Omega)$ in hypothesis (2.11)). Similarly, in view of (3.18) and (3.17), one has, for almost every $x \in \Omega$ and for every $r, r^{\prime} \in \mathbf{R}$

$$
\left\{\begin{array}{l}
\left|\frac{\partial N^{\varepsilon}}{\partial r}(x, r)-\frac{\partial N^{\varepsilon}}{\partial r}\left(x, r^{\prime}\right)\right| \leq \frac{c_{7} c_{4}}{c_{3}^{3} c_{1}^{3}}\left|r-r^{\prime}\right|+\frac{c_{2}}{c_{3}^{2} c_{1}^{2}} \omega\left(\frac{\left|r-r^{\prime}\right|}{c_{3} c_{1}}\right), \\
\frac{1}{c_{4} c_{2}} \leq \frac{\partial N^{\varepsilon}}{\partial r}(x, r) \leq \frac{1}{c_{3} c_{1}} .
\end{array}\right.
$$

Therefore one can apply Lemma 3.8 to the sequence $\frac{\partial N^{\varepsilon}}{\partial r}$.

This implies that there exists a subsequence $\varepsilon^{\prime}$ and two Carathéodory functions $N^{0}$ and $D^{0}$ such that for every $r \in \mathbf{R}$

$$
\begin{gathered}
N^{\varepsilon^{\prime}}(\cdot, r) \rightarrow N^{0}(\cdot, r) \quad \text { in } \mathcal{D}^{\prime}(\Omega) \text { weak-star, } \\
\frac{\partial N^{\varepsilon^{\prime}}}{\partial r}(\cdot, r) \rightarrow D^{0}(\cdot, r) \quad \text { in } L^{\infty}(\Omega) \text { weak-star, }
\end{gathered}
$$

where the functions $N^{0}$ and $D^{0}$ satisfy for almost every $x \in \Omega$ and for every $r, r^{\prime} \in \mathbf{R}$

$$
\left\{\begin{array}{l}
\left|N^{0}(x, r)-N^{0}\left(x, r^{\prime}\right)\right| \leq \frac{1}{c_{3} c_{1}}\left|r-r^{\prime}\right|, \\
N^{0}(x, 0)=\theta_{0}^{0}(x), \\
\left|D^{0}(x, r)-D^{0}\left(x, r^{\prime}\right)\right| \leq \frac{c_{7} c_{4}}{c_{3}^{3} c_{1}^{3}}\left|r-r^{\prime}\right|+\frac{c_{2}}{c_{3}^{2} c_{1}^{2}} \omega\left(\frac{\left|r-r^{\prime}\right|}{c_{3} c_{1}}\right), \\
\frac{1}{c_{4} c_{2}} \leq D^{0}(x, r) \leq \frac{1}{c_{3} c_{1}} .
\end{array}\right.
$$

From the mean value theorem and from (3.67), we have for some $\eta=\eta(x, r, h)$ with $0<\eta<1$

$$
\left\{\begin{array}{l}
\left|N^{\varepsilon}(x, r+h)-N^{\varepsilon}(x, r)-\frac{\partial N^{\varepsilon}}{\partial r}(x, r) h\right|=\left|\frac{\partial N^{\varepsilon}}{\partial r}(x, r+\eta h) h-\frac{\partial N^{\varepsilon}}{\partial r}(x, r) h\right| \\
\leq|h|\left(\frac{c_{7} c_{4}}{c_{3}^{3} c_{1}^{3}}|h|+\frac{c_{2}}{c_{3}^{2} c_{1}^{2}} \omega\left(\frac{|h|}{c_{1} c_{3}}\right)\right) .
\end{array}\right.
$$


Passing to the weak-star limit in $\mathcal{D}^{\prime}(\Omega)$ along the subsequence $\varepsilon^{\prime}$ implies that

$$
\left|N^{0}(x, r+h)-N^{0}(x, r)-D^{0}(x, r) h\right| \leq|h|\left(\frac{c_{7} c_{4}}{c_{3}^{3} c_{1}^{3}}|h|+\frac{c_{2}}{c_{3}^{2} c_{1}^{2}} \omega\left(\frac{|h|}{c_{1} c_{3}}\right)\right),
$$

namely that

$$
D^{0}(x, r)=\frac{\partial N^{0}}{\partial x}(x, r) \quad \text { a.e. } x \in \Omega, \forall r \in \mathbf{R} .
$$

This proves the assertions of Proposition 3.3 as far as the functions $N^{\varepsilon}$ are concerned. Similarly, in view of (3.22) and of (3.23), the Carathéodory functions $\frac{1}{\lambda^{\varepsilon}(x, r)}$ satisfy

$$
\begin{gathered}
\frac{1}{c_{2}} \leq \frac{1}{\lambda^{\varepsilon}(x, r)} \leq \frac{1}{c_{1}} \quad \text { a.e. } x \in \Omega, \forall r \in \mathbf{R}, \\
0 \leq \frac{1}{\lambda^{\varepsilon}(x, r)}-\frac{1}{\lambda^{\varepsilon}\left(x, r^{\prime}\right)} \leq \frac{1}{c_{1}^{2}} \frac{c_{7}}{c_{3} c_{1}}\left(r-r^{\prime}\right) \quad \text { a.e. } x \in \Omega, \forall r, r^{\prime} \in \mathbf{R}, r>r^{\prime} .
\end{gathered}
$$

Therefore one can apply Lemma 3.8 to the sequence $\frac{1}{\lambda^{\varepsilon}}$. This implies that there exists a further subsequence, still denoted by $\varepsilon^{\prime}$, and a Carathéodory function, that we denote by $\frac{1}{\lambda^{0}}$, such that for every $r \in \mathbf{R}$

$$
\frac{1}{\lambda^{\varepsilon^{\prime}(\cdot, r)}} \rightarrow \frac{1}{\lambda^{0}(\cdot, r)} \quad \text { in } L^{\infty}(\Omega) \text { weak-star }
$$

where the function $\frac{1}{\lambda^{0}}$ satisfies

$$
\begin{gathered}
\frac{1}{c_{2}} \leq \frac{1}{\lambda^{0}(x, r)} \leq \frac{1}{c_{1}} \quad \text { a.e. } x \in \Omega, \forall r \in \mathbf{R}, \\
0 \leq \frac{1}{\lambda^{0}(x, r)}-\frac{1}{\lambda^{0}\left(x, r^{\prime}\right)} \leq \frac{1}{c_{1}^{2}} \frac{c_{7}}{c_{3} c_{1}}\left(r-r^{\prime}\right) \quad \text { a.e. } x \in \Omega, \forall r, r^{\prime} \in \mathbf{R}, r>r^{\prime},
\end{gathered}
$$

which implies that

$$
\begin{gathered}
c_{1} \leq \lambda^{0}(x, r) \leq c_{2} \quad \text { a.e. } x \in \Omega, \forall r \in \mathbf{R}, \\
0 \leq \lambda^{0}\left(x, r^{\prime}\right)-\lambda^{0}(x, r) \leq \frac{c_{7} c_{2}^{2}}{c_{3} c_{1}^{3}}\left(r-r^{\prime}\right) \quad \text { a.e. } x \in \Omega, \forall r, r^{\prime} \in \mathbf{R}, r>r^{\prime} .
\end{gathered}
$$

This proves the assertions of Proposition 3.3 as far as the functions $\lambda^{\varepsilon}$ are concerned. Proposition 3.3 is proved.

Proof of Proposition 3.4. Since for almost every $x \in \Omega$ the function $r \rightarrow N^{0}(x, r)$ is one-to-one and strictly increasing from $\mathbf{R}$ into $\mathbf{R}$, since $N^{0}(x, 0)=\theta_{0}^{0}(x)$ and since

$$
\frac{1}{c_{4} c_{2}} \leq \frac{\partial N^{0}}{\partial r}(x, r) \leq \frac{1}{c_{3} c_{1}},
$$

its reciprocal function $s \rightarrow M^{0}(x, s)$ defined by (3.42) is one-to-one and strictly increasing from $\mathbf{R}$ onto $\mathbf{R}$ and satisfies (3.43) and (3.44), and the function $M^{0}: \Omega \times \mathbf{R} \rightarrow \mathbf{R}$ is a Carathéodory function.

Since $N^{0}\left(x, M^{0}(x, s)\right)=s$, the chain rule yields

$$
\left.\frac{\partial N^{0}}{\partial r}\left(x, M^{0}(x, s)\right) \frac{\partial M^{0}}{\partial s}(x, s)\right)=1,
$$


which implies, using (3.36) and (3.37)

$$
\left\{\begin{array}{l}
\left|\frac{\partial M^{0}}{\partial s}(x, s)-\frac{\partial M^{0}}{\partial s}\left(x, s^{\prime}\right)\right|=\frac{\left|\frac{\partial N^{0}}{\partial r}\left(x, M^{0}(x, s)\right)-\frac{\partial N^{0}}{\partial r}\left(x, M^{0}\left(x, s^{\prime}\right)\right)\right|}{\frac{\partial N^{0}}{\partial r}\left(x, M^{0}(x, s)\right) \frac{\partial N^{0}}{\partial r}\left(x, M^{0}\left(x, s^{\prime}\right)\right)} \\
\leq\left(c_{4} c_{2}\right)^{2}\left(\frac{c_{7} c_{4}}{c_{3}^{3} c_{1}^{3}}\left|M^{0}(x, s)-M^{0}\left(x, s^{\prime}\right)\right|+\frac{c_{2}}{c_{3}^{2} c_{1}^{2}} \omega\left(\frac{\left|M^{0}(x, s)-M^{0}\left(x, s^{\prime}\right)\right|}{c_{3} c_{1}}\right)\right),
\end{array}\right.
$$

which using (3.44) implies (3.45).

If we now define $\mu^{0}$ by $(\underline{3.46})$, properties (3.48) and (3.49) immediately follow from (3.38), (3.39) and (3.44). Similarly if we define $c^{0}$ by (3.47), property (3.50) immediately follows from (3.44) and (3.48), and property (3.51) immediately follows from

$$
\left\{\begin{array}{l}
\left|c^{0}(x, s)-c^{0}\left(x, s^{\prime}\right)\right| \\
\leq \frac{1}{\mu^{0}(x, s)}\left|\frac{\partial M^{0}}{\partial s}(x, s)-\frac{\partial M^{0}}{\partial s}\left(x, s^{\prime}\right)\right|+\left|\frac{\partial M^{0}}{\partial s}\left(x, s^{\prime}\right)\right| \frac{\left|\mu^{0}(x, s)-\mu^{0}\left(x, s^{\prime}\right)\right|}{\mu^{0}(x, s) \mu^{0}\left(x, s^{\prime}\right)}
\end{array}\right.
$$

and from (3.48), (3.45), (3.44) and (3.49).

Proposition 3.4 is proved.

4. Proof of the regularity Theorem 2.4. The idea of the proof is to differentiate equation (2.14) with respect to time. Setting $\frac{\partial v^{\varepsilon}}{\partial t}=w^{\varepsilon}$, and since

$$
\left\{\begin{aligned}
\frac{\partial}{\partial t}\left(\mu^{\varepsilon}\left(x, \theta^{\varepsilon}\right)\right) & =\frac{\partial \mu^{\varepsilon}}{\partial s}\left(x, \theta^{\varepsilon}\right) \frac{\partial \theta^{\varepsilon}}{\partial t}=\frac{\partial \mu^{\varepsilon}}{\partial s}\left(x, \theta^{\varepsilon}\right) \frac{\mu^{\varepsilon}\left(x, \theta^{\varepsilon}\right)}{c^{\varepsilon}\left(x, \theta^{\varepsilon}\right)}\left(\frac{\partial v^{\varepsilon}}{\partial x}\right)^{2} \\
& =\frac{\partial \mu^{\varepsilon}}{\partial s}\left(x, \theta^{\varepsilon}\right) \frac{1}{c^{\varepsilon}\left(x, \theta^{\varepsilon}\right) \mu^{\varepsilon}\left(x, \theta^{\varepsilon}\right)}\left(\sigma^{\varepsilon}\right)^{2}
\end{aligned}\right.
$$

one formally obtains

$$
\rho^{\varepsilon}(x) \frac{\partial w^{\varepsilon}}{\partial t}-\frac{\partial}{\partial x}\left(\mu\left(x, \theta^{\varepsilon}\right) \frac{\partial w^{\varepsilon}}{\partial t}\right)=\frac{\partial f^{\varepsilon}}{\partial t}+\frac{\partial}{\partial x}\left(\frac{\partial \mu^{\varepsilon}}{\partial s}\left(x, \theta^{\varepsilon}\right) \frac{1}{c^{\varepsilon}\left(x, \theta^{\varepsilon}\right)\left(\mu^{\varepsilon}\left(x, \theta^{\varepsilon}\right)\right)^{2}}\left(\sigma^{\varepsilon}\right)^{3}\right) .
$$

The right-hand side of this equation is bounded in $L^{2}\left(0, T ; H^{-1}(\Omega)\right)$ in view of hypothesis (2.30) on $\frac{\partial f^{\varepsilon}}{\partial t}$, of the a priori estimate (2.29) on $\sigma^{\varepsilon}$ and of the estimate

$$
\left|\frac{\partial \mu^{\varepsilon}}{\partial s}(x, s) \frac{1}{c^{\varepsilon}(x, s)\left(\mu^{\varepsilon}(x, s)\right)^{2}}\right| \leq \frac{c_{7}}{c_{3} c_{1}^{2}} .
$$


Using $w^{\varepsilon}$ as test function in this equation formally implies that $w^{\varepsilon}=\frac{\partial v^{\varepsilon}}{\partial t}$ is bounded in $L^{\infty}\left(0, T ; L^{2}(\Omega)\right) \cap L^{2}\left(0, T ; H_{0}^{1}(\Omega)\right)$, or more exactly in

$$
L_{l o c}^{\infty}\left(0, T ; L_{l o c}^{2}(\Omega)\right) \cap L_{l o c}^{2}\left(0, T ; H_{l o c}^{1}(\Omega)\right),
$$

since the boundary conditions on $\frac{\partial v^{\varepsilon}}{\partial t}$ only belong to (and are bounded in) $L^{2}(0, T)$, and since the initial conditions are even worse.

The above computation is formal, but we will make it rigourous below by writing the equation on the differential quotients $\partial^{h} v^{\varepsilon}$ in place of $\frac{\partial v^{\varepsilon}}{\partial t}$, and by using the function $\varphi^{2}(t) \psi^{2}(x) \partial^{h} v^{\varepsilon}$, where $\varphi$ and $\psi$ are cut-off functions, as test function in place of $\partial^{h} v^{\varepsilon}$.

Note that the proof holds true in any dimension, whenever the a priori estimate (2.29) holds true.

Let us now pass to the correct proof of Theorem 2.4

Let $\left(v^{\varepsilon}, \theta^{\varepsilon}\right)$ be a solution of (2.12) $-(2.18)$. For $h>0$, we denote by $\partial^{h} v^{\varepsilon}, \partial^{h} \theta^{\varepsilon}$ and $\partial^{h} f^{\varepsilon}$ the differential quotients

$$
\left\{\begin{array}{l}
\partial^{h} v^{\varepsilon}(t, x)=\frac{v^{\varepsilon}(t+h, x)-v^{\varepsilon}(t, x)}{h}, \\
\partial^{h} \theta^{\varepsilon}(t, x)=\frac{\theta^{\varepsilon}(t+h, x)-\theta^{\varepsilon}(t, x)}{h}, \\
\partial^{h} f^{\varepsilon}(t, x)=\frac{f^{\varepsilon}(t+h, x)-f^{\varepsilon}(t, x)}{h} .
\end{array}\right.
$$

Making the difference of equation (2.14) at time $t+h$ and at time $t$, we have

$$
\rho^{\varepsilon} \frac{\partial \partial^{h} v^{\varepsilon}}{\partial t}-\frac{\partial}{\partial x}\left(\mu^{\varepsilon}\left(x, \theta^{\varepsilon}(t+h, x)\right) \frac{\partial \partial^{h} v^{\varepsilon}}{\partial x}\right)=\partial^{h} f^{\varepsilon}+\frac{\partial}{\partial x}\left(\gamma_{h}^{\varepsilon} \frac{\partial v^{\varepsilon}}{\partial x}\right) \quad \text { in } \mathcal{D}^{\prime}(Q),
$$

where we have set

$$
\gamma_{h}^{\varepsilon}(t, x)=\frac{\mu^{\varepsilon}\left(x, \theta^{\varepsilon}(t+h, x)\right)-\mu^{\varepsilon}\left(x, \theta^{\varepsilon}(t, x)\right)}{h} .
$$

We now fix $\delta>0$ sufficiently small and define $\varphi_{\delta}(t)$ and $\psi_{\delta}(x)$ by

$$
\left\{\begin{array}{l}
\varphi_{\delta}(t)=\frac{t}{\delta} \quad \text { if } 0 \leq t \leq \delta, \quad \varphi_{\delta}(t)=1 \quad \text { if } t \geq \delta, \\
\psi_{\delta}(x)=\frac{x-a}{\delta} \quad \text { if } a \leq x \leq a+\delta, \quad \psi_{\delta}(x)=\frac{b-x}{\delta} \quad \text { if } b-\delta \leq x \leq b, \\
\psi_{\delta}(x)=1 \quad \text { if } a+\delta \leq x \leq b-\delta .
\end{array}\right.
$$


The function $\varphi_{\delta}^{2}(t) \psi_{\delta}^{2}(x) \partial^{h} v^{\varepsilon}(t, x)$ belongs to $L^{2}\left(0, T ; H_{0}^{1}(\Omega)\right) \cap H^{1}\left(0, T ; L^{2}(\Omega)\right)$ and can be used as test function in (4.1). This yields

$$
\left\{\begin{array}{l}
\frac{1}{2} \frac{d}{d t} \int_{\Omega} \rho^{\varepsilon}(x) \varphi_{\delta}^{2}(t) \psi_{\delta}^{2}(x)\left|\partial^{h} v^{\varepsilon}(t, x)\right|^{2} d x \\
+\int_{\Omega} \mu^{\varepsilon}\left(x, \theta^{\varepsilon}(t+h, x)\right) \varphi_{\delta}^{2}(t) \psi_{\delta}^{2}(x)\left|\frac{\partial \partial^{h} v^{\varepsilon}}{\partial x}(t, x)\right|^{2} d x \\
=\int_{\Omega} \rho^{\varepsilon}(x) \varphi_{\delta}(t) \frac{d \varphi_{\delta}}{d t}(t) \psi_{\delta}^{2}(x)\left|\partial^{h} v^{\varepsilon}(t, x)\right|^{2} d x \\
-\int_{\Omega} \mu^{\varepsilon}\left(x, \theta^{\varepsilon}(t+h, x)\right) \varphi_{\delta}^{2}(t) 2 \psi_{\delta}(x) \frac{d \psi_{\delta}}{d x}(x) \frac{\partial \partial^{h} v^{\varepsilon}}{\partial x}(t, x) \partial^{h} v^{\varepsilon}(t, x) d x \\
+\int_{\Omega} \partial^{h} f^{\varepsilon}(t, x) \varphi_{\delta}^{2}(t) \psi_{\delta}^{2}(x) \partial^{h} v^{\varepsilon}(t, x) d x \\
-\int_{\Omega} \gamma_{h}^{\varepsilon}(t, x) \frac{\partial v^{\varepsilon}}{\partial x}(t, x) \varphi_{\delta}^{2}(t) \psi_{\delta}^{2}(x) \frac{\partial \partial^{h} v^{\varepsilon}}{\partial x}(t, x) d x \\
-\int_{\Omega} \gamma_{h}^{\varepsilon}(t, x) \frac{\partial v^{\varepsilon}}{\partial x}(t, x) \varphi_{\delta}^{2}(t) 2 \psi_{\delta}(x) \frac{d \psi_{\delta}}{d x}(x) \partial^{h} v^{\varepsilon}(t, x) d x
\end{array}\right.
$$

Integration in time from 0 to $t$, estimates (2.2) and (2.5) on $\mu^{\varepsilon}$ and $\rho^{\varepsilon}$, and Young's inequality yield

$$
\left\{\begin{array}{l}
\frac{c_{5}}{2} \int_{\Omega} \varphi_{\delta}^{2}(t) \psi_{\delta}^{2}(x)\left|\partial^{h} v^{\varepsilon}(t, x)\right|^{2} d x \\
+c_{1} \int_{0}^{t} \int_{\Omega} \varphi_{\delta}^{2}\left(t^{\prime}\right) \psi_{\delta}^{2}(x)\left|\frac{\partial \partial^{h} v^{\varepsilon}}{\partial x}\left(t^{\prime}, x\right)\right|^{2} d x d t^{\prime} \\
\leq \frac{c_{6}}{\delta} \int_{0}^{t} \int_{\Omega}\left|\partial^{h} v^{\varepsilon}\left(t^{\prime}, x\right)\right|^{2} d x d t^{\prime} \\
+\frac{c_{1}}{4} \int_{0}^{t} \varphi_{\delta}^{2}\left(t^{\prime}\right) \psi_{\delta}^{2}(x)\left|\frac{\partial \partial^{h} v^{\varepsilon}}{\partial x}\left(t^{\prime}, x\right)\right|^{2} d x d t^{\prime}+\frac{1}{c_{1}} \frac{4 c_{2}^{2}}{\delta^{2}} \int_{0}^{t} \int_{\Omega}\left|\partial^{h} v^{\varepsilon}\left(t^{\prime}, x\right)\right|^{2} d x d t^{\prime} \\
+\int_{0}^{t} \int_{\Omega} \partial^{h} f^{\varepsilon}\left(t^{\prime}, x\right) \varphi_{\delta}^{2}\left(t^{\prime}\right) \psi_{\delta}^{2}(x) \partial^{h} v^{\varepsilon}\left(t^{\prime}, x\right) d x d t^{\prime} \\
+\frac{c_{1}}{4} \int_{0}^{t} \int_{\Omega} \varphi_{\delta}^{2}\left(t^{\prime}\right) \psi_{\delta}^{2}(x)\left|\frac{\partial \partial^{h} v^{\varepsilon}}{\partial x}\right|^{2} d x d t^{\prime}+\frac{1}{c_{1}} \int_{0}^{t} \int_{\Omega}\left|\gamma_{h}^{\varepsilon}\left(t^{\prime}, x\right)\right|^{2}\left|\frac{\partial v^{\varepsilon}}{\partial x}\left(t^{\prime}, x\right)\right|^{2} d x d t^{\prime} \\
+\frac{1}{\delta} \int_{0}^{t} \int_{\Omega}\left|\partial^{h} v^{\varepsilon}\left(t^{\prime}, x\right)\right|^{2} d x d t^{\prime}+\frac{1}{\delta} \int_{0}^{t} \int_{\Omega}\left|\gamma_{h}^{\varepsilon}\left(t^{\prime}, x\right)\right|^{2}\left|\frac{\partial v^{\varepsilon}}{\partial x}\left(t^{\prime}, x\right)\right|^{2} d x d t^{\prime} .
\end{array}\right.
$$


Let us now estimate the various terms which appear in the right-hand side of (4.3).

We first estimate the fourth term, say $I V^{\varepsilon}$, of the right-hand side of (4.3). Since by hypothesis (2.30) the functions $\frac{\partial f^{\varepsilon}}{\partial t}$ are bounded in $L^{2}\left(0, T ; H^{-1}(\Omega)\right)$, there exist functions $g^{\varepsilon} \in L^{2}(Q)$ such that

$$
\frac{\partial f^{\varepsilon}}{\partial t}=\frac{\partial g^{\varepsilon}}{\partial x}, \quad\left\|g^{\varepsilon}\right\|_{L^{2}(Q)} \leq C K^{\prime},
$$

where the constant $C$ only depends on $(b-a)$. Therefore

$$
\left\{\begin{aligned}
\partial^{h} f^{\varepsilon}\left(t^{\prime}, x\right) & =\frac{1}{h} \int_{t^{\prime}}^{t^{\prime}+h} \frac{\partial f^{\varepsilon}}{\partial t}(s, x) d s \\
& =\frac{1}{h} \int_{t^{\prime}}^{t^{\prime}+h} \frac{\partial g^{\varepsilon}}{\partial x}(s, x) d s=\frac{\partial}{\partial x}\left(\frac{1}{h} \int_{t^{\prime}}^{t^{\prime}+h} g^{\varepsilon}(s, x) d s\right) .
\end{aligned}\right.
$$

Then using integration by parts and Young's inequality we obtain

$$
\left\{\begin{aligned}
I V^{\varepsilon}= & \int_{0}^{t} \int_{\Omega} \partial^{h} f^{\varepsilon}\left(t^{\prime}, x\right) \varphi_{\delta}^{2}\left(t^{\prime}\right) \psi_{\delta}^{2}(x) \partial^{h} v^{\varepsilon}\left(t^{\prime}, x\right) d x d t^{\prime} \\
= & \int_{0}^{t} \int_{\Omega} \frac{\partial}{\partial x}\left(\frac{1}{h} \int_{t^{\prime}}^{t^{\prime}+h} g^{\varepsilon}(s, x) d s\right) \varphi_{\delta}^{2}\left(t^{\prime}\right) \psi_{\delta}^{2}(x) \partial^{h} v^{\varepsilon}\left(t^{\prime}, x\right) d x d t^{\prime} \\
= & -\int_{0}^{t} \int_{\Omega}\left(\frac{1}{h} \int_{t^{\prime}}^{t^{\prime}+h} g^{\varepsilon}(s, x) d s\right) \varphi_{\delta}^{2}\left(t^{\prime}\right) \psi_{\delta}^{2}(x) \frac{\partial \partial^{h} v^{\varepsilon}}{\partial x}\left(t^{\prime}, x\right) d x d t^{\prime} \\
& -\int_{0}^{t} \int_{\Omega}\left(\frac{1}{h} \int_{t^{\prime}}^{t^{\prime}+h} g^{\varepsilon}(s, x) d s\right) \varphi_{\delta}^{2}\left(t^{\prime}\right) 2 \psi_{\delta}(x) \frac{d \psi_{\delta}}{d x}(x) \partial^{h} v^{\varepsilon}\left(t^{\prime}, x\right) d x d t^{\prime} \\
\leq & \frac{c_{1}}{4} \int_{0}^{t} \int_{\Omega} \varphi_{\delta}^{2}\left(t^{\prime}\right) \psi_{\delta}^{2}(x)\left|\frac{\partial \partial^{h} v^{\varepsilon}}{\partial x}\left(t^{\prime}, x\right)\right|^{2} d x d t^{\prime}+\frac{1}{c_{1}} \int_{0}^{t} \int_{\Omega}\left|\frac{1}{h} \int_{t^{\prime}}^{t^{\prime}+h} g^{\varepsilon}(s, x) d s\right|^{2} d x d t^{\prime} \\
& +\frac{1}{\delta} \int_{0}^{t} \int_{\Omega}\left|\frac{1}{h} \int_{t^{\prime}}^{t^{\prime}+h} g^{\varepsilon}(s, x) d s\right|^{2} d x d t^{\prime}+\frac{1}{\delta} \int_{0}^{t} \int_{\Omega}\left|\partial^{h} v^{\varepsilon}\left(t^{\prime}, x\right)\right|^{2} d x d t^{\prime} .
\end{aligned}\right.
$$

In the last right-hand side, using Cauchy-Schwartz' inequality, the formula

$$
\int_{0}^{t} \int_{t^{\prime}}^{t^{\prime}+h}|z(s)| d s d t^{\prime} \leq h \int_{0}^{t+h}|z(s)| d s
$$

and the estimate (4.4) on $g^{\varepsilon}$, we obtain when $t+h \leq T$

$$
\left\{\begin{array}{l}
\int_{0}^{t} \int_{\Omega}\left|\frac{1}{h} \int_{t^{\prime}}^{t^{\prime}+h} g^{\varepsilon}(s, x) d s\right|^{2} d x d t^{\prime} \leq \int_{0}^{t} \int_{\Omega} \frac{1}{h^{2}}\left(h \int_{t^{\prime}}^{t^{\prime}+h}\left|g^{\varepsilon}(s, x)\right|^{2} d s\right) d x d t^{\prime} \\
\leq \int_{0}^{t+h} \int_{\Omega}\left|g^{\varepsilon}(s, x)\right|^{2} d x d s \leq C^{2} K^{\prime 2} .
\end{array}\right.
$$


In short, when $t+h \leq T$, we have proved that

$$
\left\{\begin{aligned}
I V^{\varepsilon} & \leq \frac{c_{1}}{4} \int_{0}^{t} \int_{\Omega} \varphi_{\delta}^{2}\left(t^{\prime}\right) \psi_{\delta}^{2}(x)\left|\frac{\partial \partial^{h} v^{\varepsilon}}{\partial x}\left(t^{\prime}, x\right)\right|^{2} d x d t^{\prime} \\
& +\left(\frac{1}{c_{1}}+\frac{1}{\delta}\right) C^{2} K^{\prime 2}+\frac{1}{\delta} \int_{0}^{t} \int_{\Omega}\left|\partial^{h} v^{\varepsilon}\left(t^{\prime}, x\right)\right|^{2} d x d t^{\prime} .
\end{aligned}\right.
$$

We next estimate the sixth and eighth terms of the right-hand side of (4.3). Using the definition (2.19) of $\sigma^{\varepsilon}$, the estimate (2.2) on $\mu^{\varepsilon}$, Hölder's inequality with $\frac{2}{3}+\frac{1}{3}=1$, and the a priori estimate (2.29) on $\sigma^{\varepsilon}$, we have

$$
\left\{\begin{aligned}
X^{\varepsilon} & =\int_{0}^{t} \int_{\Omega}\left|\gamma_{h}^{\varepsilon}\left(t^{\prime}, x\right)\right|^{2}\left|\frac{\partial v^{\varepsilon}}{\partial x}\left(t^{\prime}, x\right)\right|^{2} d x d t^{\prime} \\
& =\int_{0}^{t} \int_{\Omega}\left|\gamma_{h}^{\varepsilon}\left(t^{\prime}, x\right)\right|^{2}\left|\frac{1}{\mu^{\varepsilon}\left(x, \theta^{\varepsilon}\left(t^{\prime}, x\right)\right)}\right|^{2}\left|\sigma^{\varepsilon}\left(t^{\prime}, x\right)\right|^{2} d x d t^{\prime} \\
& \leq \frac{1}{c_{1}^{2}}\left(\int_{0}^{t} \int_{\Omega}\left|\gamma_{h}^{\varepsilon}\left(t^{\prime}, x\right)\right|^{3} d x d t^{\prime}\right)^{\frac{2}{3}}\left(\int_{0}^{t} \int_{\Omega}\left|\sigma^{\varepsilon}\left(t^{\prime}, x\right)\right|^{6} d x d t^{\prime}\right)^{\frac{1}{3}} \\
& \leq \frac{C^{2} C^{\star 2}}{c_{1}^{2}}\left(\int_{0}^{t} \int_{\Omega}\left|\gamma_{h}^{\varepsilon}\left(t^{\prime}, x\right)\right|^{3} d x d t^{\prime}\right)^{\frac{2}{3}} .
\end{aligned}\right.
$$

On the other hand, the definition (4.2) of $\gamma_{h}^{\varepsilon}$ and the Lipschitz continuity (2.1) of $\mu^{\varepsilon}$ with respect to $s$ yield

$$
\left\{\begin{aligned}
\left|\gamma_{t}^{\varepsilon}\left(t^{\prime}, x\right)\right| & =\left|\frac{\mu^{\varepsilon}\left(x, \theta^{\varepsilon}\left(t^{\prime}+h, x\right)\right)-\mu^{\varepsilon}\left(x, \theta^{\varepsilon}\left(t^{\prime}, x\right)\right)}{h}\right| \\
& \leq \frac{c_{7}}{h}\left|\theta^{\varepsilon}\left(t^{\prime}+h, x\right)-\theta^{\varepsilon}\left(t^{\prime}, x\right)\right| \leq \frac{c_{7}}{h}\left|\int_{t^{\prime}}^{t^{\prime}+h} \frac{\partial \theta^{\varepsilon}}{\partial t}(s, x) d s\right| .
\end{aligned}\right.
$$

But equation (2.15) on $\theta^{\varepsilon}$, definition (2.19) of $\sigma^{\varepsilon}$, and estimates (2.2) and (2.4) on $\mu^{\varepsilon}$ and $c^{\varepsilon}$ yield

$$
\left\{\begin{aligned}
\left|\frac{\partial \theta^{\varepsilon}}{\partial t}(s, x)\right| & =\frac{\partial \theta^{\varepsilon}}{\partial t}(s, x)=\frac{\mu^{\varepsilon}\left(x, \theta^{\varepsilon}(s, x)\right)}{c^{\varepsilon}\left(x, \theta^{\varepsilon}(s, x)\right)}\left|\frac{\partial v^{\varepsilon}}{\partial x}(s, x)\right|^{2} \\
& =\frac{1}{c^{\varepsilon}\left(x, \theta^{\varepsilon}(s, x)\right) \mu^{\varepsilon}\left(x, \theta^{\varepsilon}(s, x)\right)}\left|\sigma^{\varepsilon}(s, x)\right|^{2} \leq \frac{1}{c_{3} c_{1}}\left|\sigma^{\varepsilon}(s, x)\right|^{2} .
\end{aligned}\right.
$$

Finally by Hölder's inequality with $\frac{1}{3}+\frac{2}{3}=1$, we have

$$
\left|\gamma_{h}^{\varepsilon}\left(t^{\prime}, x\right)\right|^{3} \leq\left(\frac{c_{7}}{c_{3} c_{1}} \frac{1}{h} \int_{t^{\prime}}^{t^{\prime}+h}\left|\sigma^{\varepsilon}(s, x)\right|^{2} d s\right)^{3} \leq \frac{c_{7}^{3}}{c_{3}^{3} c_{1}^{3}} \frac{1}{h} \int_{t^{\prime}}^{t^{\prime}+h}\left|\sigma^{\varepsilon}(s, x)\right|^{6} d x d s .
$$


Using formula (4.5) and the a priori estimate (2.29) on $\sigma^{\varepsilon}$ we obtain when $t+h \leq T$

$$
X^{\varepsilon} \leq \frac{C^{2} C^{\star 2}}{c_{1}^{2}} \frac{c_{7}^{2}}{c_{3}^{2} c_{1}^{2}}\left(\int_{0}^{t+h} \int_{\Omega}\left|\sigma^{\varepsilon}(s, x)\right|^{6} d x d s\right)^{\frac{2}{3}} \leq \frac{c_{7}^{2}}{c_{3}^{2} c_{1}^{4}} C^{6} C^{\star 6} .
$$

We finally estimate the term

$$
\int_{0}^{t} \int_{\Omega}\left|\partial^{h} v^{\varepsilon}\left(t^{\prime}, x\right)\right|^{2} d x d t^{\prime}
$$

which appears in the first, third and seventh terms of the right-hand side of (4.3), as well as in the last term of the right-hand side of (4.6). Since

$$
\partial^{h} v^{\varepsilon}\left(t^{\prime}, x\right)=\frac{1}{h} \int_{t^{\prime}}^{t^{\prime}+h} \frac{\partial v^{\varepsilon}}{\partial t}(s, x) d s
$$

using Cauchy-Schwartz' inequality, formula (4.5) and the a priori estimate (2.21) on $\frac{\partial v^{\varepsilon}}{\partial t}$, we have when $t+h \leq T$

$$
\left\{\begin{array}{l}
\int_{0}^{t} \int_{\Omega}\left|\partial^{h} v^{\varepsilon}\left(t^{\prime}, x\right)\right|^{2} d x d t^{\prime} \leq \int_{0}^{t} \int_{\Omega} \frac{1}{h^{2}} h \int_{t^{\prime}}^{t^{\prime}+h}\left|\frac{\partial v^{\varepsilon}}{\partial t}(s, x)\right|^{2} d s d x d t^{\prime} \\
\leq \int_{0}^{t+h} \int_{\Omega}\left|\frac{\partial v^{\varepsilon}}{\partial t}(s, x)\right|^{2} d s \leq C^{\star 2} .
\end{array}\right.
$$

Using in (4.3) the estimates (4.6) and (4.7) on $I V^{\varepsilon}$ and $X^{\varepsilon}$ as well as (4.8), we have proved that when $t+h \leq T$

$$
\left\{\begin{array}{l}
\frac{c_{5}}{2} \int_{\Omega} \varphi_{\delta}^{2}(t) \psi_{\delta}^{2}(x)\left|\partial^{h} v^{\varepsilon}(t, x)\right|^{2} d x+\frac{c_{1}}{4} \int_{0}^{t} \int_{\Omega} \varphi_{\delta}^{2}\left(t^{\prime}\right) \psi_{\delta}^{2}(x)\left|\frac{\partial \partial^{h} v^{\varepsilon}}{\partial x}\left(t^{\prime}, x\right)\right|^{2} d x d t^{\prime} \\
\leq\left(\frac{c_{6}}{\delta}+\frac{4}{\delta^{2}} \frac{c_{2}^{2}}{c_{1}}+\frac{2}{\delta}\right) C^{\star 2}+\left(\frac{1}{c_{1}}+\frac{1}{\delta}\right) C^{2} K^{\prime 2}+\left(\frac{1}{c_{1}}+\frac{1}{\delta}\right) \frac{c_{7}^{2}}{c_{3}^{2} c_{1}^{4}} C^{6} C^{\star 6}
\end{array}\right.
$$

Letting $h$ tend to zero, this implies in particular that for every fixed $\delta>0$, every $\varepsilon>0$, and every $t$ with $\delta \leq t \leq T$, we have

$$
\left\{\begin{array}{l}
\frac{c_{5}}{2} \int_{\Omega_{\delta}}\left|\frac{\partial v^{\varepsilon}}{\partial t}(t, x)\right|^{2} d x+\frac{c_{1}}{4} \int_{\delta}^{T} \int_{\Omega_{\delta}}\left|\frac{\partial\left(\frac{\partial v^{\varepsilon}}{\partial t}\right)}{\partial x}\left(t^{\prime}, x\right)\right|^{2} d x d t^{\prime} \\
\leq 2\left(\left(\frac{c_{6}}{\delta}+\frac{4}{\delta^{2}} \frac{c_{2}^{2}}{c_{1}}+\frac{2}{\delta}\right) C^{\star 2}+\left(\frac{1}{c_{1}}+\frac{1}{\delta}\right) C^{2} K^{\prime 2}+\left(\frac{1}{c_{1}}+\frac{1}{\delta}\right) \frac{c_{7}^{2}}{c_{3}^{2} c_{1}^{4}} C^{6} C^{\star 6}\right),
\end{array}\right.
$$

which is nothing but (2.33).

Theorem 2.4 is proved. 
5. Proof of the homogenization Theorem 2.5. Since the data satisfy (2.1)(2.11), Theorem 2.1 implies that the unique solution $\left(v^{\varepsilon}, \theta^{\varepsilon}\right)$ of (2.12)-(2.18) satisfies estimates (2.21) - (2.22), and that the stress $\sigma^{\varepsilon}$ defined by (2.19) satisfies the a priori estimate (2.23). By Proposition 2.3 the stress $\sigma^{\varepsilon}$ also satisfies the a priori estimate (2.29).

On the other hand, the transformed temperature $\tau^{\varepsilon}$ satisfies the a priori estimate (3.34). Therefore we can extract from the subsequence $\varepsilon^{\prime}$ defined in Proposition $3.3 \mathrm{a}$ new subsequence denoted by $\varepsilon^{\prime \prime}$ such that for some $v, \theta, \sigma$ and $\tau$ we have

$$
\left\{\begin{aligned}
& v^{\varepsilon^{\prime \prime}} \rightarrow v \text { in } L^{\infty}\left(0, T ; H^{1}(\Omega)\right) \text { weak-star, } \\
& \frac{\partial v^{\varepsilon^{\prime \prime}}}{\partial t} \rightarrow \frac{\partial v}{\partial t} \quad \text { in } L^{2}\left(0, T ; L^{2}(\Omega)\right) \text { weak } \\
& \theta^{\varepsilon^{\prime \prime}} \rightarrow \theta \quad \text { in } \mathcal{D}^{\prime}(Q) \text { weak-star, } \\
& \sigma^{\varepsilon^{\prime \prime}} \rightarrow \sigma \text { in } L^{\infty}\left(0, T ; L^{2}(\Omega)\right) \text { weak-star, } \\
& \quad \text { and in } L^{2}\left(0, T ; H^{1}(\Omega)\right) \cap L^{6}(Q) \text { weak } \\
& \tau^{\varepsilon^{\prime \prime}} \rightarrow \tau \quad \text { in } L^{1}(Q) \text { strong }
\end{aligned}\right.
$$

where for the strong convergence of $\tau^{\varepsilon^{\prime \prime}}$ we have used the fact that the embedding of $W^{1,1}(Q)$ in $L^{1}(Q)$ is compact.

We will prove that $(v, \theta)$ is a solution of (2.12)-(2.18) for the viscosity function $\mu^{0}$ and the heat coefficient $c^{0}$ defined in Proposition 3.4, and for the density $\rho^{0}$ and the data $f^{0}, v_{a}^{0}, v_{b}^{0}, v_{0}^{0}$ and $\theta_{0}^{0}$ defined in (2.6)-(2.11). Since this solution is unique, this will prove that it is not necessary to extract a subsequence $\varepsilon^{\prime \prime}$ from the sequence $\varepsilon^{\prime}$ and that the whole sequences $v^{\varepsilon^{\prime}}, \theta^{\varepsilon^{\prime}}$ and $\sigma^{\varepsilon^{\prime}}$ converge, as stated in Theorem 2.5] and Remark 2.6.

5.1. First case: the case where $\frac{\partial f^{\varepsilon}}{\partial t}$ is bounded in $L^{2}\left(0, T ; H^{-1}(\Omega)\right)$. In this first case we assume that, in addition to the hypotheses of Theorem 2.1 hypothesis (2.34) also holds true. Then Theorem 2.4 implies that $\frac{\partial v^{\varepsilon}}{\partial t}$ is bounded in $L_{l o c}^{2}\left(0, T ; H_{l o c}^{1}(\Omega)\right.$ ) (see (2.33)), namely that

$$
\frac{\partial^{2} v^{\varepsilon}}{\partial x \partial t} \quad \text { is bounded in } L_{l o c}^{2}(Q) \text {. }
$$

Then the definition (2.19) of $\sigma^{\varepsilon}$, namely

$$
\sigma^{\varepsilon}(t, x)=\mu^{\varepsilon}\left(x, \theta^{\varepsilon}(t, x)\right) \frac{\partial v^{\varepsilon}}{\partial x}(t, x),
$$


and equation (2.15) on $\theta^{\varepsilon}$ imply that

$$
\left\{\begin{aligned}
\frac{\partial \sigma^{\varepsilon}}{\partial t} & =\mu^{\varepsilon}\left(x, \theta^{\varepsilon}\right) \frac{\partial^{2} v^{\varepsilon}}{\partial x \partial t}+\frac{\partial \mu^{\varepsilon}}{\partial s}\left(x, \theta^{\varepsilon}\right) \frac{\partial \theta^{\varepsilon}}{\partial t} \frac{\partial v^{\varepsilon}}{\partial x} \\
& =\mu^{\varepsilon}\left(x, \theta^{\varepsilon}\right) \frac{\partial^{2} v^{\varepsilon}}{\partial x \partial t}+\frac{\partial \mu^{\varepsilon}}{\partial s}\left(x, \theta^{\varepsilon}\right) \frac{1}{c^{\varepsilon}\left(x, \theta^{\varepsilon}\right)} \mu^{\varepsilon}\left(x, \theta^{\varepsilon}\right)\left(\frac{\partial v^{\varepsilon}}{\partial x}\right)^{2} \frac{\partial v^{\varepsilon}}{\partial x} \\
& =\mu^{\varepsilon}\left(x, \theta^{\varepsilon}\right) \frac{\partial^{2} v^{\varepsilon}}{\partial x \partial t}+\frac{\partial \mu^{\varepsilon}}{\partial s}\left(x, \theta^{\varepsilon}\right) \frac{1}{c^{\varepsilon}\left(x, \theta^{\varepsilon}\right)\left(\mu^{\varepsilon}\left(x, \theta^{\varepsilon}\right)\right)^{2}}\left(\sigma^{\varepsilon}\right)^{3}
\end{aligned}\right.
$$

Using the estimates (2.2), (2.1) and (2.4) on $\mu^{\varepsilon}$, $\frac{\partial \mu^{\varepsilon}}{\partial s}$, and $c^{\varepsilon}$, and the estimates (15.2) and (2.29) on $\frac{\partial^{2} v^{\varepsilon}}{\partial x \partial t}$ and $\sigma^{\varepsilon}$, we conclude that

$$
\frac{\partial \sigma^{\varepsilon}}{\partial t} \text { is bounded in } L_{l o c}^{2}(Q) \text {. }
$$

Combined with the a priori estimate (2.23) on $\sigma^{\varepsilon}$, this implies that

$$
\sigma^{\varepsilon} \quad \text { is bounded in } H_{l o c}^{1}(Q) \text {. }
$$

Turning back to (5.1) and using Rellich's compactness theorem, we have proved that

$$
\sigma^{\varepsilon^{\prime \prime}} \rightarrow \sigma \quad \text { in } L_{l o c}^{2}(Q) \quad \text { strong. }
$$

By the definition (3.21) of the function $\lambda^{\varepsilon}$, the definition (3.20) of the transformed temperature $\tau^{\varepsilon}$ and the definition (2.19) of the stress $\sigma^{\varepsilon}$, we have (see (3.24) )

$$
\sigma^{\varepsilon}=\lambda^{\varepsilon}\left(x, \tau^{\varepsilon}\right) \frac{\partial v^{\varepsilon}}{\partial x}
$$

or equivalently, in view of the estimates (3.22) on $\lambda^{\varepsilon}$

$$
\frac{\partial v^{\varepsilon}}{\partial x}=\frac{1}{\lambda^{\varepsilon}\left(x, \tau^{\varepsilon}\right)} \sigma^{\varepsilon} \quad \text { in } \mathcal{D}^{\prime}(Q)
$$

Since by Proposition 3.3 the functions $\frac{1}{\lambda^{\varepsilon^{\prime}}(x, r)}$, which are uniformly (in $x$ and $\varepsilon$ ) Lipschitz continuous in $s$ (see $(\underline{3.23)})$, converge in $L^{\infty}(\Omega)$ weak-star to $\frac{1}{\lambda^{0}(x, r)}$ for every $r$ fixed (see (3.41) ), and since $\tau^{\varepsilon^{\prime \prime}}$ converges to $\tau$ strong in $L^{1}(\Omega)$ (see (5.1)), the second part of Lemma 3.8 implies that

$$
\frac{1}{\lambda^{\varepsilon^{\prime \prime}}\left(x, \tau^{\varepsilon^{\prime \prime}}(t, x)\right)} \rightarrow \frac{1}{\lambda^{0}(x, \tau(t, x))} \text { in } \mathcal{D}^{\prime}(Q) \text { weak-star }
$$

and also in $L^{\infty}(Q)$ weak-star since the sequence $\frac{1}{\lambda^{\varepsilon}\left(x, \tau^{\varepsilon}\right)}$ is bounded in $L^{\infty}(Q)$. Combined with the strong convergence of $\sigma^{\varepsilon^{\prime \prime}}$ in $L_{l o c}^{2}(Q)$ (see (15.3)), this allows us to pass to the limit in (5.4). We have proved that

$$
\frac{\partial v}{\partial x}=\frac{1}{\lambda^{0}(x, \tau)} \sigma \quad \text { in } \mathcal{D}^{\prime}(Q)
$$


or equivalently that

$$
\sigma=\lambda^{0}(x, \tau) \frac{\partial v}{\partial x} \quad \text { in } \mathcal{D}^{\prime}(Q) .
$$

On the other hand, the definition (3.20) of the transformed temperature $\tau^{\varepsilon}$ asserts that

$$
\theta^{\varepsilon}(t, x)=N^{\varepsilon}\left(x, \tau^{\varepsilon}(t, x)\right) .
$$

Since by Proposition 3.3 the functions $N^{\varepsilon^{\prime}}(x, r)$, which are uniformly (in $x$ and $\varepsilon$ ) Lipschitz continuous in $s$ (see (3.17)), converge to $N^{0}(x, r)$ in $\mathcal{D}^{\prime}(\Omega)$ weak-star for every $r$ fixed, since the functions $\theta_{0}^{\varepsilon}$ and $\theta_{0}^{0}$ satisfy (2.11), and finally since $\tau^{\varepsilon^{\prime \prime}}$ converges strong to $\tau$ in $L^{1}(Q)$, the second part of Lemma 3.8 implies that

$$
N^{\varepsilon^{\prime \prime}}\left(x, \tau^{\varepsilon^{\prime \prime}}(t, x)\right) \rightarrow N^{0}(x, \tau(t, x)) \text { in } \mathcal{D}^{\prime}(Q) \text { weak-star. }
$$

Therefore passing to the limit in (5.6) with the help of (5.1), we have proved that

$$
\theta(t, x)=N^{0}(x, \tau(t, x)) .
$$

By the definition (3.42) of the reciprocal function $M^{0}$ of the function $N^{0}$, this is equivalent to

$$
\tau(t, x)=M^{0}(x, \theta(t, x)) .
$$

In view of the definition (3.46) of the function $\mu^{0}$, (5.5) and (5.7) imply that

$$
\sigma=\mu^{0}(x, \theta) \frac{\partial v}{\partial x} .
$$

It is now easy to pass to the limit in equation (2.14). Since

$$
\rho^{\varepsilon}(x) \frac{\partial v^{\varepsilon}}{\partial t}=\frac{\partial}{\partial t}\left(\rho^{\varepsilon}(x) v^{\varepsilon}\right)
$$

and since $v^{\varepsilon^{\prime \prime}}$ tends to $v$ in $L^{2}(Q)$ strong by (5.1) and Rellich's compactness theorem, one obtains, in view of (2.6), (5.1) and (2.7)

$$
\rho^{0} \frac{\partial v}{\partial t}-\frac{\partial \sigma}{\partial x}=f^{0} \quad \text { in } \mathcal{D}^{\prime}(Q)
$$

which, in view of formula (5.8) for $\sigma$, is nothing but equation (2.14) with $\varepsilon=0$.

Similarly, since $\sigma^{\varepsilon^{\prime \prime}}$ converges to $\sigma$ in $L_{l o c}^{2}(Q)$ strong (see (5.3)), one easily passes to the limit in equation (3.29), namely (see (3.25))

$$
\frac{\partial \tau^{\varepsilon}}{\partial t}=\left|\sigma^{\varepsilon}\right|^{2} \quad \text { in } \mathcal{D}^{\prime}(Q) \text {. }
$$

This yields

$$
\frac{\partial \tau}{\partial t}=|\sigma|^{2} \quad \text { in } \mathcal{D}^{\prime}(Q) .
$$

But (5.7) and the definition (3.47) of $c^{0}(x, s)$ in Proposition 3.4 imply that

$$
\left\{\begin{aligned}
\frac{\partial \tau}{\partial t}(t, x) & =\frac{\partial}{\partial t}\left(M^{0}(x, \theta(t, x))\right)=\frac{\partial M^{0}}{\partial s}(x, \theta(t, x)) \frac{\partial \theta}{\partial t}(t, x) \\
& =c^{0}(x, \theta(t, x)) \mu^{0}(x, \theta(t, x)) \frac{\partial \theta}{\partial t}(t, x),
\end{aligned}\right.
$$


which, combined with equation (5.9), formula (5.8) for $\sigma$ and the fact that $\mu^{0}$ is strictly positive (see (3.48)), implies that

$$
c^{0}(x, \theta) \frac{\partial \theta}{\partial t}=\mu^{0}(x, \theta)\left(\frac{\partial v}{\partial x}\right)^{2} \quad \text { in } \mathcal{D}^{\prime}(Q),
$$

i.e. equation (2.15) with $\varepsilon=0$.

We observe (see (5.1)) that $v$ enjoys the regularity (2.12). We now investigate the boundary and initial conditions on $v$. Passing to the limit in (2.16) with the help of (5.1) and (2.8) implies that $v$ satisfies

$$
v(t, a)=v_{a}^{0}(t), \quad v(t, b)=v_{b}^{0}(t) \quad \text { a.e. } t \in(0, T),
$$

i.e. the boundary conditions (2.16) with $\varepsilon=0$.

For what concerns the initial condition on $v$, we define for every fixed $\varphi \in C_{c}^{\infty}(\Omega)$ the functions $\mathcal{V}^{\varepsilon}:(0, T) \rightarrow \mathbf{R}$ by

$$
\mathcal{V}^{\varepsilon}(t)=\int_{\Omega} v^{\varepsilon}(t, x) \varphi(x) d x \quad \text { a.e. } t \in(0, T),
$$

and the function $\mathcal{V}:(0, T) \rightarrow \mathbf{R}$ by

$$
\mathcal{V}(t)=\int_{\Omega} v(t, x) \varphi(x) d x \quad \text { a.e. } t \in(0, T) .
$$

In view of (5.1), the functions $\mathcal{V}^{\varepsilon}$ satisfy

$$
\mathcal{V}^{\varepsilon^{\prime \prime}} \rightarrow \mathcal{V} \quad \text { in } H^{1}(0, T) \text { weak, }
$$

and therefore that

$$
\mathcal{V}^{\varepsilon^{\prime \prime}} \rightarrow \mathcal{V} \quad \text { in } C^{0}([0, T]) \text { strong. }
$$

This implies in particular that

$$
\mathcal{V}^{\varepsilon^{\prime \prime}}(0)=\int_{\Omega} v^{\varepsilon^{\prime \prime}}(0, x) \varphi(x) d x \rightarrow \mathcal{V}(0)=\int_{\Omega} v(0, x) \varphi(x) d x,
$$

and since $v^{\varepsilon}(0, x)=v_{0}^{\varepsilon}(x)$ (see (2.17) ), this yields in view of (2.9)

$$
v(0, x)=v_{0}^{0}(x) \text { a.e. } x \in \Omega,
$$

i.e. the initial condition (2.17) with $\varepsilon=0$.

It remains to prove that $\theta$ enjoys the regularity (2.13) and satisfies the initial condition (2.18) with $\varepsilon=0$.

For that, using equation (2.15) on $\theta^{\varepsilon}$ and the definition (2.19) of $\sigma^{\varepsilon}$, we write

$$
\frac{\partial \theta^{\varepsilon}}{\partial t}=\frac{1}{c^{\varepsilon}\left(x, \theta^{\varepsilon}\right)} \mu^{\varepsilon}\left(x, \theta^{\varepsilon}\right)\left(\frac{\partial v^{\varepsilon}}{\partial x}\right)^{2}=\frac{1}{c^{\varepsilon}\left(x, \theta^{\varepsilon}\right) \mu^{\varepsilon}\left(x, \theta^{\varepsilon}\right)}\left(\sigma^{\varepsilon}\right)^{2},
$$

which, in view of the estimates (2.2) and (2.4) on $\mu^{\varepsilon}$ and $c^{\varepsilon}$ and of the a priori estimate (2.29) on $\sigma^{\varepsilon}$, implies that

$$
\frac{\partial \theta^{\varepsilon}}{\partial t} \quad \text { is bounded in } L^{3}(Q) \text {. }
$$

Combined with (5.1), this implies that

$$
\frac{\partial \theta}{\partial t} \in L^{3}(Q)
$$


We now define for every fixed $\varphi \in C_{c}^{\infty}(\Omega)$ the functions $\mathcal{T}^{\varepsilon}:(0, T) \rightarrow \mathbf{R}$ by

$$
\mathcal{T}^{\varepsilon}(t)=\int_{\Omega} \theta^{\varepsilon}(t, x) \varphi(x) d x \quad \text { a.e. } t \in(0, T),
$$

and the function $\mathcal{T}:(0, T) \rightarrow \mathbf{R}$ by

$$
\mathcal{T}(t)=\int_{\Omega} \theta(t, x) \varphi(x) d x \quad \text { a.e. } t \in(0, T) .
$$

In view of (5.10), one has

$$
\frac{\partial \mathcal{T}^{\varepsilon}}{\partial t} \text { bounded in } L^{3}(0, T)
$$

and in view of the initial condition (2.18) on $\theta^{\varepsilon}$ one has

$$
\mathcal{T}^{\varepsilon}(0)=\int_{\Omega} \theta^{\varepsilon}(0, x) \varphi(x) d x=\int_{\Omega} \theta_{0}^{\varepsilon}(x) \varphi(x) d x .
$$

Using estimate (2.11) on $\theta_{0}^{\varepsilon}$, we deduce that $\mathcal{T}^{\varepsilon}(0)$ is bounded and therefore that $\mathcal{T}^{\varepsilon}$ is bounded in $W^{1,3}(0, T)$. Since $\mathcal{T}^{\varepsilon^{\prime \prime}}$ converge to $\mathcal{T}$ in $\mathcal{D}^{\prime}(0, T)$ weak-star in view of (5.1), this implies that

$$
\mathcal{T}^{\varepsilon^{\prime \prime}} \rightarrow \mathcal{T} \quad \text { in } W^{1,3}(0, T) \text { weak }
$$

and therefore that

$$
\mathcal{T}^{\varepsilon^{\prime \prime}} \rightarrow T \quad \text { in } C^{0}([0, T]) \text { strong. }
$$

This implies in particular that

$$
\mathcal{T}^{\varepsilon^{\prime \prime}}(0)=\int_{\Omega} \theta^{\varepsilon}(0, x) \varphi(x) d x \rightarrow \mathcal{T}(0)=\int_{\Omega} \theta(0, x) \varphi(x) d x .
$$

Using (5.12) and the convergence (2.11) on $\theta_{0}^{\varepsilon}$, we conclude that

$$
\theta(0, x)=\theta_{0}^{0}(x) \quad \text { a.e. } x \in \Omega,
$$

i.e. the initial condition (2.18) with $\varepsilon=0$.

Since $\frac{\partial \theta}{\partial t}$ belongs to $L^{3}(Q)$ and since $\theta(0, x)$ belongs to $L^{1}(\Omega)$, the distribution $\theta$ actually belongs to $W^{1,3}\left(0, T ; L^{1}(\Omega)\right)$, and therefore to $W^{1,1}\left(0, T ; L^{1}(\Omega)\right)$. This proves that $\theta$ enjoys the regularity (2.13).

In conclusion, we have proved that $v, \theta$ and $\sigma$ defined in (5.1) satisfy (2.12)-(2.18) and (2.19) with $\varepsilon=0$. Uniqueness of the solution of this system implies that $v=v^{0}$, $\theta=\theta^{0}$ and $\sigma=\sigma^{0}$, and that the convergences (5.1) take place for the whole subsequence $\varepsilon^{\prime}$ defined in Proposition 3.3 and not only for a further subsequence $\varepsilon^{\prime \prime}$.

Theorem 2.5) is therefore proved when hypothesis (2.34) holds true.

5.2. Second case: the case where $f^{\varepsilon}$ is compact in $L^{2}(Q)$. In this second case we assume that (2.35) holds true.

Let $\psi$ be a given function such that

$$
\psi \in C_{c}^{\infty}(\mathbf{R}), \quad \psi \geq 0, \quad \int_{\mathbf{R}} \psi(t) d t=1
$$

We define the regularizing sequence $\psi_{\eta}$ by

$$
\psi_{\eta}(t)=\frac{1}{\eta} \psi\left(\frac{t}{\eta}\right), \quad \eta>0 .
$$


Next we consider the functions $f_{\eta}^{\varepsilon}$ and $f_{\eta}^{0}$ defined by

$$
\begin{aligned}
f_{\eta}^{\varepsilon}(t, x) & =\int_{\mathbf{R}} \tilde{f}^{\varepsilon}(s, x) \psi_{\eta}(t-s) d s, \\
f_{\eta}^{0}(t, x) & =\int_{\mathbf{R}} \tilde{f}^{0}(s, x) \psi_{\eta}(t-s) d s,
\end{aligned}
$$

where $\tilde{f}^{\varepsilon}$ and $\tilde{f}^{0}$ are defined by extending $f^{\varepsilon}$ and $f^{0}$ by zero to $\mathbf{R} \times \Omega$, i.e.

$$
\begin{aligned}
& \left\{\begin{array}{l}
\tilde{f}^{\varepsilon}(t, x)=f^{\varepsilon}(t, x) \quad \text { if } \quad 0<t<T, \\
\tilde{f}^{\varepsilon}(t, x)=0 \text { if } t<0 \quad \text { or if } t>T,
\end{array}\right. \\
& \left\{\begin{array}{l}
\tilde{f}^{0}(t, x)=f^{0}(t, x) \text { if } 0<t<T, \\
\tilde{f}^{0}(t, x)=0 \text { if } t<0 \text { or if } t>T .
\end{array}\right.
\end{aligned}
$$

The functions $f_{\eta}^{\varepsilon}$ satisfy

$$
\left\{\begin{array}{l}
\left\|f_{\eta}^{\varepsilon}\right\|_{L^{2}(Q)} \leq K \\
\left\|\frac{\partial f_{\eta}^{\varepsilon}}{\partial t}\right\|_{L^{2}(Q)} \leq \frac{K\left\|\psi^{\prime}\right\|_{L^{1}(\mathbf{R})}}{\eta} \\
f_{\eta}^{\varepsilon} \rightarrow f_{\eta}^{0} \quad \text { in } L^{2}(Q) \text { weak for } \eta \text { fixed } \\
f_{\eta}^{0} \rightarrow f^{0} \quad \text { in } L^{2}(Q) \text { strong. }
\end{array}\right.
$$

Let $\left(v_{\eta}^{\varepsilon}, \theta_{\eta}^{\varepsilon}\right)$ be the unique solution of (2.12)-(2.18) for the external forces $f_{\eta}^{\varepsilon}$, the coefficients $\mu^{\varepsilon}, c^{\varepsilon}$ and $\rho^{\varepsilon}$ and the data $v_{a}^{\varepsilon}, v_{b}^{\varepsilon}, v_{0}^{\varepsilon}$ and $\theta_{0}^{\varepsilon}$. Since the second assertion of (5.13) ensures that for $\eta>0$ fixed, $\frac{\partial f_{\eta}^{\varepsilon}}{\partial t}$ is bounded in $L^{2}\left(0, T ; H^{-1}(\Omega)\right)$, the result proved in the previous subsection implies that for $\eta>0$ fixed one has

$$
\left\{\begin{array}{l}
v_{\eta}^{\varepsilon^{\prime}} \rightarrow v_{\eta}^{0} \quad \text { in } L^{\infty}\left(0, T ; H^{1}(\Omega)\right) \cap H^{1}\left(0, T ; L^{2}(\Omega)\right) \text { weak-star } \\
\theta_{\eta}^{\varepsilon^{\prime}} \rightarrow \theta_{\eta}^{0} \quad \text { in } \mathcal{D}^{\prime}(Q) \text { weak-star }
\end{array}\right.
$$

where the subsequence $\varepsilon^{\prime}$ is defined in Proposition 3.3. and where $\left(v_{\eta}^{0}, \theta_{\eta}^{0}\right)$ is the unique solution of (2.12)-(2.18) for the external forces $f_{\eta}^{0}$, the coefficients $\mu^{0}, c^{0}$ and $\rho^{0}$ and the data $v_{a}^{0}, v_{b}^{0}, v_{0}^{0}$ and $\theta_{0}^{0}$.

On the other hand, since $\left\|f_{\eta}^{\varepsilon}-f_{\eta}^{0}\right\|_{L^{2}(Q)} \leq\left\|f^{\varepsilon}-f^{0}\right\|_{L^{2}(Q)}$, we have for every $\varepsilon>0$ and every $\eta>0$

$$
\left\{\begin{aligned}
\left\|f_{\eta}^{\varepsilon}-f^{\varepsilon}\right\|_{L^{2}(Q)} & \leq\left\|f_{\eta}^{\varepsilon}-f_{\eta}^{0}\right\|_{L^{2}(Q)}+\left\|f_{\eta}^{0}-f^{0}\right\|_{L^{2}(Q)}+\left\|f^{0}-f^{\varepsilon}\right\|_{L^{2}(Q)} \\
& \leq 2\left\|f^{\varepsilon}-f^{0}\right\|_{L^{2}(Q)}+\left\|f_{\eta}^{0}-f^{0}\right\|_{L^{2}(Q)} .
\end{aligned}\right.
$$


Since $\left(v_{\eta}^{\varepsilon}, \theta_{\eta}^{\varepsilon}\right)$ and $\left(v^{\varepsilon}, \theta^{\varepsilon}\right)$ respectively correspond to the data $\left(f_{\eta}^{\varepsilon}, v_{a}^{\varepsilon}, v_{b}^{\varepsilon}, v_{0}^{\varepsilon}, \theta_{0}^{\varepsilon}\right)$ and $\left(f^{\varepsilon}, v_{a}^{\varepsilon}, v_{b}^{\varepsilon}, v_{0}^{\varepsilon}, \theta_{0}^{\varepsilon}\right)$, the a priori estimate (2.24) implies that

$$
\left\{\begin{array}{l}
\left\|v_{\eta}^{\varepsilon}-v^{\varepsilon}\right\|_{L^{\infty}\left(0, T ; L^{2}(\Omega)\right)}+\left\|v_{\eta}^{\varepsilon}-v^{\varepsilon}\right\|_{L^{2}\left(0, T ; H^{1}(\Omega)\right)}+\left\|\theta_{\eta}^{\varepsilon}-\theta^{\varepsilon}\right\|_{L^{\infty}\left(0, T ; L^{1}(\Omega)\right)} \\
\leq C^{\star \star}\left\|f_{\eta}^{\varepsilon}-f^{\varepsilon}\right\|_{L^{2}(Q)},
\end{array}\right.
$$

where $C^{\star \star}$ is a constant which depends only on $c_{1}, c_{2}, c_{3}, c_{4}, c_{5}, c_{6}, c_{7},(b-a)$ and $K$. Therefore we have

$$
\left\{\begin{array}{l}
\left\|v_{\eta}^{\varepsilon}-v^{\varepsilon}\right\|_{L^{\infty}\left(0, T ; L^{2}(\Omega)\right)}+\left\|v_{\eta}^{\varepsilon}-v^{\varepsilon}\right\|_{L^{2}\left(0, T ; H^{1}(\Omega)\right)}+\left\|\theta_{\eta}^{\varepsilon}-\theta^{\varepsilon}\right\|_{L^{\infty}\left(0, T ; L^{1}(\Omega)\right)} \\
\leq C^{\star \star}\left(2\left\|f^{\varepsilon}-f^{0}\right\|_{L^{2}(Q)}+\left\|f_{\eta}^{0}-f^{0}\right\|_{L^{2}(Q)}\right) .
\end{array}\right.
$$

For $\left(v_{\eta}^{0}, \theta_{\eta}^{0}\right)$ and $\left(v^{0}, \theta^{0}\right)$, the same a priori estimate (2.24) implies that

$$
\left\{\begin{array}{l}
\left\|v_{\eta}^{0}-v^{0}\right\|_{L^{\infty}\left(0, T ; L^{2}(\Omega)\right)}+\left\|v_{\eta}^{0}-v^{0}\right\|_{L^{2}\left(0, T ; H^{1}(\Omega)\right)}+\left\|\theta_{\eta}^{0}-\theta^{0}\right\|_{L^{\infty}\left(0, T ; L^{1}(\Omega)\right)} \\
\leq C^{\star \star}\left\|f_{\eta}^{0}-f^{0}\right\|_{L^{2}(Q)} .
\end{array}\right.
$$

We now write

$$
\left\{\begin{array}{l}
v^{\varepsilon}-v^{0}=v^{\varepsilon}-v_{\eta}^{\varepsilon}+v_{\eta}^{\varepsilon}-v_{\eta}^{0}+v_{\eta}^{0}-v^{0}, \\
\theta^{\varepsilon}-\theta^{0}=\theta^{\varepsilon}-\theta_{\eta}^{\varepsilon}+\theta_{\eta}^{\varepsilon}-\theta_{\eta}^{0}+\theta_{\eta}^{0}-\theta^{0} .
\end{array}\right.
$$

Combining (5.17) with (5.15), (5.14), (5.16) and (5.13) proves that, for the subsequence $\varepsilon^{\prime}$ defined in Proposition 3.3.

$$
\begin{cases}v^{\varepsilon^{\prime}} \rightarrow v^{0} & \text { in } L^{\infty}\left(0, T ; L^{2}(\Omega)\right) \cap L^{2}\left(0, T ; H^{1}(\Omega)\right) \text { weak-star, } \\ \theta^{\varepsilon^{\prime}} \rightarrow \theta^{0} & \text { in } \mathcal{D}^{\prime}(Q) \text { weak-star, }\end{cases}
$$

where $\left(v^{0}, \theta^{0}\right)$ is the solution of (2.12)-(2.18) for the coefficients $\mu^{0}, c^{0}$ and $\rho^{0}$ and for the data $\left(f^{0}, v_{a}^{0}, v_{b}^{0}, v_{0}^{0}, \theta_{0}^{0}\right)$. Since $v^{\varepsilon}$ is bounded in $L^{\infty}\left(0, T ; H^{1}(\Omega)\right) \cap H^{1}\left(0, T ; L^{2}(\Omega)\right)$ (see (2.21) ), the convergence of $v^{\varepsilon^{\prime}}$ also takes place in $L^{\infty}\left(0, T ; H^{1}(\Omega)\right) \cap H^{1}\left(0, T ; L^{2}(\Omega)\right)$ weak-star. This proves convergences (2.36), (2.37) and (2.38). Convergence (2.39) is then proved using (5.4) as in the previous subsection.

Theorem 2.5 is therefore proved when hypothesis (2.35) holds true.

\section{An example showing that the homogenized heat coefficient $c^{0}$ depends} in general on the temperature. In this section we consider the case of a material made of layers of size $\varepsilon$ of some given homogeneous phases characterized by densities $\rho_{i}(x)=\rho_{i}$, viscosity functions $\mu_{i}(x, s)=\mu_{i}(s)$ and heat coefficients $c_{i}(x, s)=c_{i}(s)$, $i=1, \ldots, I$. In this special case we give explicit formulas for the homogenized density $\rho_{0}$, the homogenized viscosity function $\mu_{0}$ and the homogenized heat coefficient $c_{0}$. 
In this setting, the density, the viscosity function and the heat coefficient are given by

$$
\left\{\begin{array}{l}
\rho^{\varepsilon}(x)=\sum_{i=1}^{I} \rho_{i} \chi_{i}^{\varepsilon}(x), \\
\mu^{\varepsilon}(x, s)=\sum_{i=1}^{I} \mu_{i}(s) \chi_{i}^{\varepsilon}(x), \\
c^{\varepsilon}(x, s)=\sum_{i=1}^{I} c_{i}(s) \chi_{i}^{\varepsilon}(x) .
\end{array}\right.
$$

Here, for every fixed $\varepsilon>0$, the functions $\chi_{i}^{\varepsilon}(x)$ are characteristic functions of disjoint measurable sets whose union is $\Omega$, i.e. functions which satisfy

$$
\chi_{i}^{\varepsilon}(x)\left(1-\chi_{i}^{\varepsilon}(x)\right)=0 \quad \text { for } i=1, \ldots, I, \quad \sum_{i=1}^{I} \chi_{i}^{\varepsilon}(x)=1 \quad \text { a.e. } x \in \Omega,
$$

(this setting generalizes the case of a material made of layers of size $\varepsilon$ ), the constants $\rho_{i}$ satisfy for $i=1, \ldots, I$

$$
c_{5} \leq \rho_{i} \leq c_{6},
$$

the functions $\mu_{i}: \mathbf{R} \rightarrow \mathbf{R}$ are bounded, Lipschitz continuous, nonincreasing functions which satisfy for $i=1, \ldots, I$

$$
-c_{7} \leq \frac{\partial \mu_{i}}{\partial s}(s) \leq 0, \quad c_{1} \leq \mu_{i}(s) \leq c_{2} \quad \forall s \in \mathbf{R},
$$

and finally the functions $c_{i}: \mathbf{R} \rightarrow \mathbf{R}$ are bounded, uniformly continuous functions which satisfy for $i=1, \ldots, I$

$$
\left|c_{i}(s)-c_{i}\left(s^{\prime}\right)\right| \leq \omega\left(\left|s-s^{\prime}\right|\right), \quad c_{3} \leq c_{i}(s) \leq c_{4} \quad \forall s, s^{\prime} \in \mathbf{R},
$$

where $\omega$ is a modulus of continuity. Then the functions $\rho^{\varepsilon}(x), \mu^{\varepsilon}(x, s)$ and $c^{\varepsilon}(x, s)$ defined by (6.1) satisfy hypotheses (2.1)-(2.5).

We moreover assume that for $i=1, \ldots, I$

$$
\chi_{i}^{\varepsilon} \rightarrow p_{i} \text { in } L^{\infty}(\Omega) \text { weak-star, }
$$

where the function $p_{i}$ describes the volume fraction of the material $i$ at the point $x$ in the homogenized material. Under this hypothesis, the extraction of a subsequence $\varepsilon^{\prime}$ in Proposition 3.3 and in the homogenization Theorem 2.5 is no longer necessary. Note that

$$
0 \leq p_{i}(x) \leq 1 \quad \text { for } i=1, \ldots, I, \quad \sum_{i=1}^{I} p_{i}(x)=1 \quad \text { a.e. } x \in \Omega .
$$

Let us give in this case explicit formulas for the functions $\rho^{0}, \mu^{0}$ and $c^{0}$ which appear in the homogenization Theorem 2.5 .

The homogenized density $\rho^{0}$ is given by (see (2.6) $)$

$$
\rho^{0}(x)=\sum_{i=1}^{I} \rho_{i} p_{i}(x) \quad \text { a.e. } x \in \Omega .
$$

Considering for simplicity the case where

$$
\theta_{0}^{\varepsilon}(x)=0,
$$


and defining, for $I=1, \ldots, I$, the functions $M_{i}: \mathbf{R} \rightarrow \mathbf{R}$ by

$$
M_{i}(s)=\int_{0}^{s} c_{i}\left(s^{\prime}\right) \mu_{i}\left(s^{\prime}\right) d s^{\prime}, \quad \forall s \in \mathbf{R},
$$

as well as their inverse functions $N_{i}: \mathbf{R} \rightarrow \mathbf{R}$ by

$$
M_{i}(s)=r \quad \Longleftrightarrow \quad s=N_{i}(r) \quad \forall s, r \in \mathbf{R},
$$

we have (see (3.10) and (3.14)

$$
\begin{array}{ll}
M^{\varepsilon}(x, s)=\sum_{i=1}^{I} M_{i}(s) \chi_{i}^{\varepsilon}(x) \quad \text { a.e. } x \in \Omega, \forall s \in \mathbf{R}, \\
N^{\varepsilon}(x, r)=\sum_{i=1}^{I} N_{i}(r) \chi_{i}^{\varepsilon}(x), \quad \text { a.e. } x \in \Omega, \forall r \in \mathbf{R} .
\end{array}
$$

Then the function $N^{0}$ defined by (3.40) is given by

$$
N^{0}(x, r)=\sum_{i}^{I} N_{i}(r) p_{i}(x) .
$$

The function $M^{0}(x, s)$ is then defined as the reciprocal function of $N^{0}(x, r)$ (see (3.42)), i.e.

$$
M^{0}(x, s)=r \quad \Longleftrightarrow \quad N^{0}(x, r)=s \quad \text { a.e. } x \in \Omega, \forall s, r \in \mathbf{R} .
$$

Defining, for $i=1, \ldots, I$, the functions $\lambda_{i}: \mathbf{R} \rightarrow \mathbf{R}$ by

$$
\lambda_{i}(r)=\mu_{i}\left(N_{i}(r)\right), \quad \forall r \in \mathbf{R},
$$

we have (see (3.21))

$$
\lambda^{\varepsilon}(x, r)=\mu^{\varepsilon}\left(x, N^{\varepsilon}(x, r)\right)=\sum_{i=1}^{I} \lambda_{i}(r) \chi_{i}^{\varepsilon}(x) \quad \text { a.e. } x \in \Omega, \forall r \in \mathbf{R} .
$$

Then the homogenized function $\lambda^{0}$ defined by (3.41) is given by

$$
\frac{1}{\lambda^{0}(x, r)}=\sum_{i=1}^{I} \frac{p_{i}(x)}{\lambda_{i}(r)}
$$

Finally, the homogenized viscosity function $\mu^{0}$ and the homogenized heat coefficient $c^{0}$ defined by (3.46) and (3.47) are given by

$$
\begin{gathered}
\mu^{0}\left(x, N^{0}(x, r)\right)=\lambda^{0}(x, r), \\
\left\{\begin{aligned}
c^{0}\left(x, N^{0}(x, r)\right) & =\frac{\partial M^{0}}{\partial s}\left(x, N^{0}(x, r)\right) \frac{1}{\mu_{0}\left(x, N^{0}(x, r)\right)} \\
& =\frac{\partial M^{0}}{\partial s}\left(x, N^{0}(x, r)\right) \frac{1}{\lambda_{0}(x, r)} .
\end{aligned}\right.
\end{gathered}
$$

We deduce from (6.10) and (6.9) that

$$
\mu^{0}\left(x, N^{0}(x, r)\right)=\left(\sum_{i=1}^{I} \frac{p_{i}(x)}{\lambda_{i}(r)}\right)^{-1} .
$$


On the other hand, using the chain rule formula in $r=M_{i}\left(N_{i}(r)\right)$ and the definitions (6.5) and (6.8) of $M_{i}$ and $\lambda_{i}$, we have for $i=1, \ldots, I$

$$
1=\frac{\partial M_{i}}{\partial s}\left(N_{i}(r)\right) \frac{\partial N_{i}}{\partial r}(r)=c_{i}\left(N_{i}(r)\right) \mu_{i}\left(N_{i}(r)\right) \frac{\partial N_{i}}{\partial r}(r)=c_{i}\left(N_{i}(r)\right) \lambda_{i}(r) \frac{\partial N_{i}}{\partial r}(r) .
$$

In view of (6.7), this yields

$$
\frac{\partial N^{0}}{\partial r}(x, r)=\sum_{i=1}^{I} \frac{\partial N_{i}}{\partial r}(r) p_{i}(x)=\sum_{i=1}^{I} \frac{p_{i}(x)}{c_{i}\left(N_{i}(r)\right) \lambda_{i}(r)} .
$$

Similarly, using the chain rule formula in the identity $M^{0}\left(x, N^{0}(x, r)\right)=r$, we have

$$
\left.\frac{\partial M^{0}}{\partial s}\left(x, N^{0}(x, r)\right) \frac{\partial N^{0}}{\partial r}(x, r)\right)=1,
$$

and therefore using (6.11) and (6.14), and then (6.13) and (6.9)

$$
c^{0}\left(x, N^{0}(x, r)\right)=\frac{1}{\frac{\partial N^{0}}{\partial r}(x, r)} \frac{1}{\lambda_{0}(x, r)}=\left(\sum_{i=1}^{I} \frac{p_{i}(x)}{c_{i}\left(N_{i}(r)\right) \lambda_{i}(r)}\right)^{-1}\left(\sum_{i=1}^{I} \frac{p_{i}(x)}{\lambda_{i}(r)}\right) .
$$

In this particular setting of a material made of layers of homogeneous phases, we have proved the following result.

THEOREM 6.1 (Explicit formulas for a material made of layers of homogeneous phases). When $\rho^{\varepsilon}, \mu^{\varepsilon}$ and $c^{\varepsilon}$ are defined by (6.1), and when (6.2) and (6.4) are satisfied, Propositions 3.3 and 3.4 and Theorem 2.5 hold true for the whole sequence $\varepsilon$ and for the functions $\mu^{0}$ and $c^{0}$ given by formulas (6.12) and (6.15), where the function $N^{0}(x, r)$ is defined by formula (6.7).

We now claim that in general the homogenized heat coefficient $c^{0}$ does depend on $s$, i.e. on the temperature, even if the heat coefficients of each phase do not depend on it, i.e. even if for $i=1, \ldots, I$

$$
c_{i}(s)=c_{i} \in \mathbf{R} \quad \forall s \in \mathbf{R} .
$$

Indeed in the special case where (6.16) holds true, formula (6.15) reads as

$$
c^{0}\left(x, N^{0}(x, r)\right)=\left(\sum_{i=1}^{I} \frac{p_{i}(x)}{c_{i} \lambda_{i}(r)}\right)^{-1}\left(\sum_{i=1}^{I} \frac{p_{i}(x)}{\lambda_{i}(r)}\right) .
$$

When all the $c_{i}$ are all equal to some $c^{\star}$, i.e. when $c^{\varepsilon}(x, s)=c^{\star}$ is fixed independently of $x$ and of $s$, then formula (6.17) implies that $c^{0}(x, s)=c^{\star}$, and of course the homogenized heat coefficient does not depend on the temperature in this case. But when the $c_{i}$ are different, i.e. when the $c^{\varepsilon}(x, s)$ do oscillate, then the heat coefficient $c^{0}(x, s)$ in general depends on $s$.

Let us first consider the example of the mixture of two phases. Then $c^{0}\left(x, N^{0}(x, r)\right)$ reads as

$$
c^{0}\left(x, N^{0}(x, r)\right)=\frac{\frac{p_{1}(x)}{\lambda_{1}(r)}+\frac{p_{2}(x)}{\lambda_{2}(r)}}{\frac{p_{1}(x)}{c_{1} \lambda_{1}(r)}+\frac{p_{2}(x)}{c_{2} \lambda_{2}(r)}}=\frac{\frac{p_{1}(x)}{p_{2}(x)}+\frac{\lambda_{1}(r)}{\lambda_{2}(r)}}{\frac{1}{c_{2}}\left(\frac{c_{2}}{c_{1}} \frac{p_{1}(x)}{p_{2}(x)}+\frac{\lambda_{1}(r)}{\lambda_{2}(r)}\right)} .
$$

When $\lambda_{1}(r) / \lambda_{2}(r)$ is not constant, the right-hand side of (6.18), and therefore its lefthand side, depends on $r$. Then $c^{0}(x, s)$ depends on $s$. 
In the general case of $I$ phases with $I>2$, the function $c^{0}\left(x, N^{0}(x, r)\right)$ also depends on $r$ in general. Let us consider for simplicity the case where the volume functions $p_{i}(x)$ do not depend on $x$, i.e. the case where $p_{i}(x)=p_{i}$ for $i=1, \ldots, I$. Set for $i=1, \ldots, I$

$$
z_{i}(r)=\frac{p_{i}}{c_{i} \lambda_{i}(r)}
$$

Then $c^{0}\left(x, N^{0}(x, r)\right)$ does not depend on $x$ and reads as

$$
c^{0}\left(x, N^{0}(x, r)\right)=\frac{\sum_{i=1}^{I} c_{i} z_{i}(r)}{\sum_{i=1}^{I} z_{i}(r)} .
$$

Therefore the function $c^{0}\left(x, N^{0}(x, r)\right)$ does not depend on $r$ if and only if there exists a constant $c^{\star}$ such that

$$
\sum_{i=1}^{I} c_{i} z_{i}(r)=c^{\star} \sum_{i=1}^{I} z_{i}(r),
$$

i.e. if and only if

$$
\sum_{i=1}^{I}\left(c_{i}-c^{\star}\right) z_{i}(r)=0 .
$$

When the functions $z_{i}, i=1, \ldots, I$, are linearly independent, this is never the case except when $c_{i}=c^{\star}$ for $i=1, \ldots, I$, i.e. when $c^{\varepsilon}(x, s)=c^{\star}$ is fixed independently of $x$ and of $s$.

Acknowledgements. The results of the present work were obtained during visits made by the first author to the Laboratoire Jacques-Louis Lions, whose support and hospitality are gratefully acknowledged. The support of the European RTD project Lifebelt IST-2001-38165 is also gratefully acknowledged. Finally the interest and support of UIK and UMT are sincerely acknowledged.

\section{REFERENCES}

[1] J. Aboudi, M. Pindera, and S.M. Arnold, Higher-order theory for functionally graded materials, Composite Part B (Engineering) 30 (1999), 777-832.

[2] J. Aboudi, M. Pindera, and S.M. Arnold, Higher-order theory for periodic multiphase materials with inelastic phases, Int. J. Plasticity 19 (2003), 805-847.

[3] Y. Bansal and M.J. Pindera, A second look at the higher-order theory for periodic multiphase materials, J. Appl. Mech. 72 (2005), 177-195.

[4] R.C. Batra and B.M. Love, Adiabatic shear bands in functionally graded materials, J. Thermal Stresses 27 (2004), 1101-1123.

[5] T. Baxevanis, T. Katsaounis, and A. Tzavaras, A finite element method for computing shear band formation, in Proceedings of the International Hyperbolic Conference (Osaka 2004), Yokohama Publ., Yokohama, to appear.

[6] A. Bensoussan, J.-L. Lions, and G. Papanicolaou, Asymptotic analysis for periodic structures, North-Holland, Amsterdam, 1978. MR0503330 (82h:35001)

[7] N. Charalambakis and F. Murat, Weak solutions to initial-boundary value problem for the shearing of non-homogeneous thermoviscoplastic materials, Proc. Royal Soc. Edinburgh 113A (1989), 257265. MR.1037731 (91b:73008)

[8] N. Charalambakis and F. Murat, Approximation by finite elements, existence and uniqueness for a model of stratified thermoviscoplastic materials, Ric. Mat. 55 (2006), to appear. 
[9] C.M. Dafermos and L. Hsiao, Adiabatic shearing of incompressible fluids with temperaturedependent viscosity, Quart. Appl. Math. 41 (1983), 45-58. MR0700660 (84g:76026)

[10] J. Hodowany, G. Ravichandran, A.J. Rosakis, and P. Rosakis, Partition of plastic work into heat and stored energy in metals, J. Exp. Mech. 40 (2000), 113-123.

[11] Z.H. Jin and R.C. Batra, Some basic fracture mechanics concepts in functionally graded materials, J. Mech. Phys. Solids 44 (1996), 1221-1235.

[12] P. Rosakis, A.J. Rosakis, G. Ravichandran, and J. Hodowany, A thermodynamical internal variable model for the partition of plastic work into heat and stored energy in metals, J. Mech. Phys. Solids 48 (2000), 582-607.

[13] P. Rosakis, A.J. Rosakis, G. Ravichandran, and J. Hodowany, On the conversion of plastic work into heat during high-strain-rate deformation, AIP Conference Proceedings 620 (2002), 557-562.

[14] E. Sanchez-Palencia, Nonhomogeneous media and vibration theory, Lecture Notes in Physics, vol. 127, Springer Verlag, Berlin, Heidelberg, 1978. MR0578345 (82j:35010)

[15] L. Tartar, Homogénéisation et compacité par compensation, Cours Peccot, Collège de France, Paris, March 1977. Partly written in: F. Murat, $H$-convergence, Séminaire d'Analyse Fonctionnelle et Numérique 1977-1978, Université d'Alger, Alger, multicopied, 34 pages. English translation: F. Murat and L. Tartar, $H$-convergence, in Topics in the mathematical modelling of composite materials, ed. by A. Cherkaev and R.V. Kohn, Progress in Nonlinear Differential Equations and their Applications, vol. 31, Birkhäuser, Boston, 1997, pp. 21-43. MR0557520 (81c:73012)

[16] A. Tzavaras, Shearing of materials exhibiting thermal softening or temperature dependent viscosity, Quart. Appl. Math. 44 (1986), 1-12. MR0840438 (87m:76007)

[17] A. Tzavaras, Plastic shearing of materials exhibiting strain hardening or strain softening, Arch. Rat. Mech. Anal. 94 (1986), 39-58. MR0831769 (87e:73054) 